\title{
Supersymmetric monojets at the Large Hadron Collider
}

\author{
Benjamin C. Allanach, ${ }^{a}$ Sebastian $\mathrm{Grab}^{b}$ and Howard E. Haber ${ }^{b}$ \\ ${ }^{a}$ DAMTP, CMS, University of Cambridge, \\ Wilberforce Road, Cambridge, CB3 0WA, U.K. \\ ${ }^{b}$ Department of Physics and SCIPP, University of California, \\ 1156 High Street, Santa Cruz, CA 95064, U.S.A. \\ E-mail: b.c.allanach@damtp.cam.ac.uk, sgrab@scipp.ucsc.edu, \\ haber@scipp.ucsc.edu
}

ABSTRACT: Supersymmetric monojets may be produced at the Large Hadron Collider by the process $q g \rightarrow \widetilde{q} \widetilde{\chi}_{1}^{0} \rightarrow q \widetilde{\chi}_{1}^{0} \widetilde{\chi}_{1}^{0}$, leading to a jet recoiling against missing transverse momentum. We discuss the feasibility and utility of the supersymmetric monojet signal. In particular, we examine the possible precision with which one can ascertain the $\widetilde{\chi}_{1}^{0} \widetilde{q} q$ coupling via the rate for monojet events. Such a coupling contains information on the composition of the $\widetilde{\chi}_{1}^{0}$ and helps bound dark matter direct detection cross-sections and the dark matter relic density of the $\widetilde{\chi}_{1}^{0}$. It also provides a check of the supersymmetric relation between gauge couplings and gaugino-quark-squark couplings.

Keywords: Supersymmetry Phenomenology

ARXiv EPRINT: 1010.4261 


\section{Contents}

1 Introduction $\quad 1$

2 Supersymmetric monojets revisited 3

2.1 Associated production of squarks and neutralinos 3

$\begin{array}{ll}2.2 & \text { The nature of the LSP }\end{array}$

3 Testing the supersymmetric coupling relations with monojets $\quad 8$

$\begin{array}{lll}3.1 & \text { The signal process } & 8\end{array}$

$\begin{array}{ll}3.2 & \text { Major backgrounds and basic cuts } \\ \end{array}$

4 Measurement of the $\widetilde{\chi}_{1}^{0} \widetilde{q} q$ coupling for a bino LSP 12

$\begin{array}{ll}4.1 \text { Benchmark scenario } & 12\end{array}$

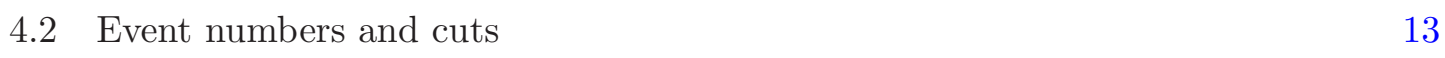

$\begin{array}{lll}4.3 & \text { Coupling reconstruction } & 16\end{array}$

5 Reconstruction of the $\widetilde{\chi}_{1}^{0} \widetilde{q} q$ coupling for a wino LSP $\quad 18$

$\begin{array}{ll}5.1 \text { Benchmark scenario } & 18\end{array}$

$\begin{array}{lll}5.2 & \text { Event numbers and cuts } & 18\end{array}$

$\begin{array}{ll}5.3 \text { Coupling reconstruction } & 21\end{array}$

5.4 Reconstruction of couplings under the assumption of a wino LSP 23

$\begin{array}{llr}6 & \text { Summary and conclusion } & 26\end{array}$

$\begin{array}{ll}\text { A The Jacobian peak in the transverse momentum distribution } & 27\end{array}$

\section{Introduction}

With the first Large Hadron Collider (LHC) experimental run now underway, searches for physics beyond the Standard Model will begin to test new theories of TeV-scale phenomena in parameter regimes that were not accessible to previous collider facilities. There are many proposals for theories of TeV-scale physics beyond the Standard Model. These include supersymmetry, technicolor, little Higgs models, extra-dimensions, low-scale quantum gravity, etc., all of which posit the existence of new classes of fundamental particles [1]. In many such models, the lightest member of the new class is absolutely stable due to the presence of a conserved discrete symmetry. As a result, production of the lightest new physics particle (either directly or at the end of chain of heavier decaying particles) will lead to missing transverse energy signatures at the LHC. More generally, signatures from different models of $\mathrm{TeV}$-scale physics often possess similar features, and distinguishing among model interpretations may be quite challenging. 
In this paper, we assume that after a number of years of LHC running, a class of new particles has been discovered. We assume that their masses and Standard Model quantum numbers will be relatively well determined. We also assume that we will have some information about the spins of the new particles. Under these assumptions, how strong will the case be for a supersymmetric (SUSY) interpretation of the new physics?

Ideally, one would first try to identify the new particles as superpartners of Standard Model particles. Such an attempt will likely be incomplete, as the entire spectrum of the new physics may not have been revealed, and the spins of the new particles may not be reliably known in all cases. In isolation, the discovery of a neutral color octet fermion or a color triplet scalar does not necessarily imply the discovery of the gluino and the squark. Due to supersymmetry-breaking, the masses of the new particles would not provide any evidence for a supersymmetric interpretation. However, supersymmetry-breaking effects will typically have a small impact on dimension-four couplings [2]. These couplings therefore reflect the underlying supersymmetric structure. Hence, the measurement of a relation among couplings could provide very strong evidence for a supersymmetric interpretation of the new physics.

In the supersymmetric extension of the Standard Model, the couplings of gluons to squark pairs and gluino pairs and the couplings of the photon, $W^{ \pm}$and $Z$ to squark pairs and slepton pairs are governed by $\mathrm{SU}(3) \times \mathrm{SU}(2) \times \mathrm{U}(1)$ gauge invariance. In contrast, supersymmetry relates the gauge couplings of particles (or their supersymmetric partners) to the Yukawa couplings of gauginos to particle-sparticle pairs. Similarly, supersymmetry relates the Yukawa couplings of Higgs bosons to the Yukawa couplings of higgsinos to particle-sparticle pairs. Thus, the precision measurement of the interaction strengths of particle-sparticle vertices can provide the smoking gun for a supersymmetric interpretation of the new physics.

Precision measurements of couplings is usually the domain of $e^{+} e^{-}$colliders. Indeed, there is a rich program of precision supersymmetry proposed for an experimental program at the ILC [3]. However, until the ILC becomes a reality (current projections suggest that this is unlikely during the present decade), we must rely on the LHC running at high luminosity to provide the necessary data for interpreting the fundamental nature of new physics discoveries. Ultimately, a combined LHC/ILC analysis would yield the most precise tests for the supersymmetric interpretation of new physics [4].

Until now, only a few analyses have been proposed for the LHC. In ref. [5], same flavor squark pair production at the LHC was investigated in order to measure the strength of the gluino-squark-quark coupling. But this analysis still relied on additional data from a linear collider. The analysis was refined in ref. [6] by taking into account more production channels of strongly interacting SUSY particles. It was found that a test of the SUSY relation for the gluino-squark-quark operator based solely on LHC data might be possible with a precision better than $10 \%$. A later study [7] assuming $300 \mathrm{fb}^{-1}$ of LHC data, with varying degrees of ILC input, found similar fractional precisions of 3-7\%. Finally, in ref. [8], (left-handed) squark pair production was considered via color-singlet gaugino exchange in the $t$ - and $u$-channel. It was shown that a measurement of this process, and therefore a measurement of the wino-squark-quark coupling, might be possible at the LHC. 
We propose the development of a program of precision measurements of new particle interactions at the LHC in order to provide definitive evidence in support of a possible supersymmetric interpretation of $\mathrm{TeV}$-scale physics. Such a program must necessarily comprise a broad class of new particle signatures. Ultimately, a global fit to a plethora of supersymmetric observables will be required to provide the maximal coverage of the underlying parameter space. In this paper, we take the first step by identifying a particular signature that is sensitive to the squark-quark-gaugino coupling.

In section 2, we identify the supersymmetric monojet signal as a promising arena for measuring the squark-quark-gaugino coupling. Two basic supersymmetric scenarios are considered, which depend on the identity of the lightest supersymmetric particle (LSP). We contrast the phenomenology of a bino-LSP and a wino-LSP, which arise in many models of $\mathrm{TeV}$-scale supersymmetry. In section 3 , we outline the process by which one can test the supersymmetric coupling relations at the LHC. These procedures are then employed in sections 4 and 5 to measure the squark-quark-gaugino couplings in the case of the bino-LSP and wino-LSP, respectively. Finally, we state our conclusions in section 6 . The $p_{T}$-spectrum of the monojet signal exhibits a peak at a characteristic value of the transverse momentum that is approximately given by

$$
\left(p_{T}\right)_{\text {peak }} \simeq \frac{m_{\tilde{q}}^{2}-m_{\tilde{\chi}_{1}^{0}}^{2}}{2 m_{\tilde{q}}}
$$

This result, which is derived in appendix A [cf. eq. (A.50)], is analogous to the Jacobian peak of the electron transverse momentum in $W$ production and decay.

\section{Supersymmetric monojets revisited}

\subsection{Associated production of squarks and neutralinos}

Supersymmetric monojet signatures have an interesting history. In the early 1980s, CERN $p \bar{p}$ collisions resulting in single jets ("monojet") in association with missing transverse energy in the UA1 experiment [9] were famously interpreted in terms of supersymmetric particle production [10-26]. One example of such a process was squark photino production, followed by decay of the squark into a quark and photino. Later, it was calculated that the monojet events would predict more di-jet missing transverse momentum events than were observed [27, 28]. Finally, backgrounds (particularly $W$ production, where $W \rightarrow \tau \nu_{\tau}$, followed by $\tau \rightarrow \nu_{\tau} j$ ), and $Z j$ production, where $Z \rightarrow \nu \bar{\nu}$ [29], were seen to adequately account for the monojet events.

Several authors have discussed the ability of LHC experiments to discover large extra dimensions through the monojets signal $[30,31]$ predicted by the production of a hard gluon and a Kaluza-Klein tower of gravitons. Obvious Standard Model (SM) backgrounds in the LHC environment include di-jet production, where one of the jets is lost, and the $W j, Z j$ backgrounds mentioned above. We wish to measure the rate of the dominant SM background (i.e. monojet production via $Z j$ ) by measuring the cases where $Z \rightarrow \ell^{+} \ell^{-}$ $(\ell=e, \mu)$ and using them to predict the background process. Isolated lepton vetoes help 

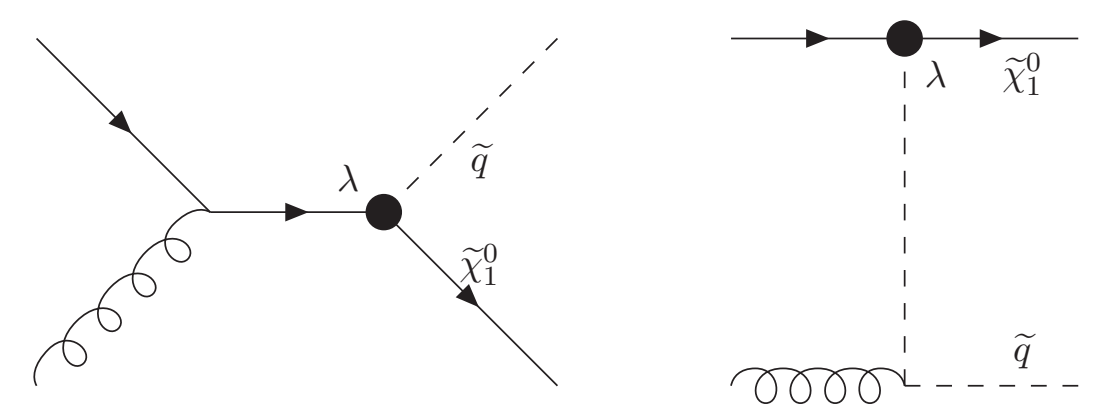

Figure 1. Tree-level Feynman diagrams leading to $q g \rightarrow \widetilde{q} \widetilde{\chi}_{1}^{0}$. Monojet signatures result from $\widetilde{q} \rightarrow q \widetilde{\chi}_{1}^{0}$.

discriminate against $W j$, and a large missing $p_{T}$ cut discriminates against the QCD dijet background. More recently, unparticle-jet production, leading to a monojet signature has also been examined [32]. The monojet $p_{T}$ spectrum is fairly featureless in either the large extra dimensional case or in the unparticle-jet case, although the rates are in general different.

Early $\sqrt{s}=7 \mathrm{TeV}$ LHC collisions resulting in monojets recorded by ATLAS [33] have already been made public. The data show no excess over SM Monte Carlo predictions for $70 \mathrm{nb}^{-1}$ of LHC data, but we find that the monojet channel is not yet sensitive to SUSY models that are not already ruled out by other experiments. ${ }^{1}$ Much more data will be required.

We wish to appraise the ability of the LHC to measure SUSY monojet production, assuming the minimal supersymmetric standard model (MSSM) via the process $q g \rightarrow \widetilde{q} \widetilde{\chi}_{1}^{0}$. At tree-level, there are two diagrams that contribute, which are exhibited in figure 1 . The tree-level scattering amplitudes for $q g \rightarrow \widetilde{q} \widetilde{\chi}_{1}^{0}$ were obtained in ref. [35, 36]. Strong SUSYQCD corrections and the leading logarithmic electroweak corrections at one loop have been treated in ref. [37] (although we note that the one-loop QCD corrections to $q g \rightarrow \widetilde{q} \widetilde{\chi}_{1}^{0}$ do not yet appear in the literature).

The processes depicted in figure 1 do not constitute the best SUSY search channel, since the production amplitude is proportional to a weak $(\lambda)$ times a strong gauge coupling. Di-squark, di-gluino and/or gluino-squark production are expected to have much higher rates because their amplitudes are proportional to the strong gauge coupling squared. This is probably why the associated production of squarks and neutralinos has not been extensively examined in the literature. On the other hand, if the processes in figure 1 could be identified and the rates measured, an estimate for the coupling $\lambda$ may result. This coupling contains information on the identity of the lightest neutralino $\widetilde{\chi}_{1}^{0}$, which may be dominantly bino, wino, higgsino or a mixture. For example, in the bino (wino) dominated case, supersymmetry dictates that $\lambda$ is proportional to $g^{\prime}(g)$, the gauge coupling of the gauge group $\mathrm{U}(1)_{Y}\left(\mathrm{SU}(2)_{L}\right)$, respectively (where the proportionality constant is fixed by supersymmetry). If one knew the constitution of the neutralino from other measurements, then the measurement of the coupling $\lambda$ in associated squark-neutralino production would

\footnotetext{
${ }^{1}$ Note, however, that multijets plus $\not{ }_{T}$ channels are already becoming competitive with published Tevatron bounds in certain corners of MSSM parameter space [34].
} 
(a)

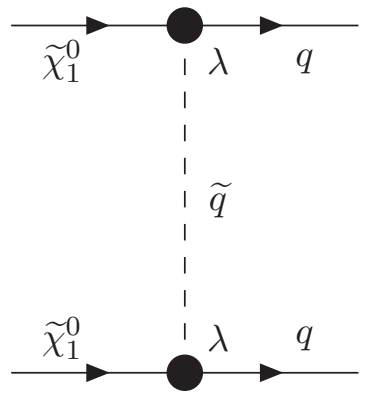

(b)

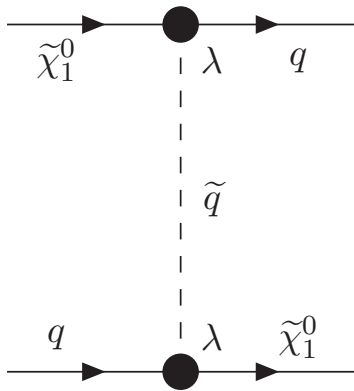

Figure 2. Tree-level Feynman diagrams contributing to (a) dark matter annihilation and (b) dark matter direct detection.

provide a direct test of supersymmetry. ${ }^{2}$

It has long been postulated that $\widetilde{\chi}_{1}^{0}$ constitutes the dark matter of our universe. Knowing its couplings is a vital ingredient in calculating how much of it is left as a thermal relic in the universe. For example, neutralinos may annihilate in the early universe through the process depicted in figure $2 \mathrm{a}$ before freezing out. Such a process involves the same vertex as the one in our signal. If $\widetilde{\chi}_{1}^{0}$ constitutes the dark matter of the universe, direct detection experiments have the chance to measure it through it causing nuclear recoils. As depicted in figure $2 \mathrm{~b}$, nuclear recoil mediated via squark exchange could contribute a significant part of the direct detection cross-section [38]. The measurement of $\lambda$ would bound the direct detection cross-section contribution from such a channel.

There are advantages and disadvantages to the SUSY monojet signature as compared to monojets resulting from large extra dimensions or unparticle-jet production. As we shall illustrate below, SUSY monojet production has features in the $p_{T}$ spectrum, which would help to convince us that the distribution is not merely an incomplete understanding of SM backgrounds. In particular, the location of the Jacobian peak provides an indication of the masses of the squark and neutralino (an analytical approximation is provided in appendix A). The disadvantage is that SUSY backgrounds may be problematic. Other SUSY processes than those in figure 1 leading to the monojet signature include: $\widetilde{\chi}_{1}^{0} \widetilde{\chi}_{1}^{0}$ production, with an initial-state radiated jet, and $\widetilde{q} \widetilde{q}$ production, where each $\widetilde{q} \rightarrow q \widetilde{\chi}_{1}^{0}$, but both quarks are either in the same direction, or one is lost (for example because it is emitted at high rapidity). The case where both quarks are in the same direction can be discriminated against with a maximum jet mass cut.

Various papers have examined SUSY monojets recently, but these are all different processes from the one we attempt to isolate and would be classified as SUSY backgrounds by our study. In ref. [39], a scenario where gluinos and neutralinos are quasi mass-degenerate were considered. Such a scenario allows a gravitino dark matter candidate while being compatible with high reheating temperatures required by thermal leptogenesis. This scenario leads to an effective monojet signature via $\widetilde{q g}$ production, where the gluinos decay to soft QCD radiation and quasi-stable neutralinos, and the squark decays to a jet and a neutralino. Weak gaugino pair production plus a jet, leading to monojet signatures, has

\footnotetext{
${ }^{2}$ As emphasized in section 1, the measurement of the gluon couplings to gluino pairs or to squark pairs does not constitute a test of supersymmetry, as these latter couplings are governed by QCD.
} 
recently been examined in ref. [40] for the case of quasi-degenerate gauginos, so that the visible products of their decays are too soft to be detected. In ref. [41], SUSY monojet signatures were examined for a region of the MSSM consistent with baryogenesis and dark matter constraints. These monojet signatures originated from stop pair production plus an additional QCD jet, where the stops decay invisibly into a soft charm quark and a $\widetilde{\chi}_{1}^{0}$. Monojet searches at the Tevatron have recently been used to place bounds on dark matter direct detection rates [42-44] and on indirect dark matter searches via gamma ray lines [45]. The collider process investigated in ref. [42-44] consisted of initial state radiation in dark matter pair production, which is classified as a background in the present paper. It was found that for very light dark matter, below $5 \mathrm{GeV}$, the inferred Tevatron bounds are stronger than those from direct detection. ${ }^{3}$ Pair production of dark matter particles in association with one or more jets at Tevatron and LHC, was also considered in ref. [46]. For our study, all processes mentioned above in this paragraph are classified as SUSY backgrounds. An earlier work [47] included predictions for monojet signatures from ultra-light gravitinos at the Tevatron and LHC. This is a different scenario from the one of interest in the present paper.

LHC and Tevatron cross sections for total SUSY monojet production were listed in ref. [48], which was primarily concerned with the case of massless $\widetilde{\chi}_{1}^{0}$ particles. Of course, the total SUSY monojet production includes our signal process (alongside SUSY backgrounds). We extend this study in several ways: for example, we perform a more detailed analysis, finding reasonable cuts to discriminate signal from background and presenting the kinematics of the events. We also discuss background subtraction. We focus on the case of massive $\widetilde{\chi}_{1}^{0}$ particles and determine with what accuracy $\lambda$ might be measured.

\subsection{The nature of the LSP}

Supersymmetric collider signals are notoriously complicated and depend strongly upon the parameter space, even if one restricts oneself to the MSSM. The monojet signal of interest for this paper depends primarily on two properties of the supersymmetric model: (i) the precise nature of the LSP (e.g., the relative contributions of the bino, wino and higgsino components of the neutralino LSP wave function); and (ii) the branching ratio of the squark into a quark and the LSP.

In $R$-parity-conserving supersymmetric models, the most likely candidate for the LSP (excluding the gravitino which is not relevant for this discussion) is the lightest neutralino $\widetilde{\chi}_{1}^{0} \cdot{ }^{4}$ In general, $\widetilde{\chi}_{1}^{0}$ is a linear combination of bino, wino and higgsino interaction eigenstates. The relative contributions of each of these components depends on the parameters that govern the neutralino mass matrix. These include $m_{Z}$, the higgsino mass parameter $\mu$, the gaugino mass parameters $M_{1}, M_{2}$, and the ratio of Higgs vacuum expectation values $\tan \beta$. (Approximate formulae for the neutralino masses and mixing matrix in terms of these parameters can be found in ref. [53].) If the higgsino component were dominant,

\footnotetext{
${ }^{3}$ Although the absence of direct detection signals is always subject to a potentially large systematic of unknown density of dark matter at the site of the experiment.

${ }^{4}$ Cosmological and laboratory constraints rule out other possible candidates such as the sneutrino [49], gluino $[50,51]$ or charged slepton [52] in almost all possible parameter regimes.
} 
then the monojet signal proposed in this paper would not be viable, as the corresponding cross-section for squark-neutralino production would be suppressed by a light quark Yukawa coupling. In this case, other methods must be employed to test for supersymmetric coupling relations. However, we note that in many (though not all) models of supersymmetry, the parameter $|\mu|$ is parametrically larger than $M_{1}, M_{2}$ and $m_{Z}$, in which case the higgsino component of the LSP is small. Henceforth, we will assume that the underlying SUSY model supports a $\widetilde{\chi}_{1}^{0}$ that is dominantly gaugino in nature.

This still leaves the question of the relative contributions of the bino and wino components of the $\widetilde{\chi}_{1}^{0}$ wave function. In any supersymmetric model with the unification of tree-level gaugino mass parameters, the ratio of the low-energy values of the gaugino mass parameters is given by [54],

$$
M_{1} \simeq \frac{5 g^{\prime 2}}{3 g^{2}} M_{2} \simeq 0.5 M_{2}
$$

Assuming that the tree-level gaugino masses are non-vanishing (and are of order $m_{Z}$ ), then the bino component of the $\widetilde{\chi}_{1}^{0}$ wave function is dominant. This is typical of most mSUGRA models [54], but is more general and depends only on the assumptions outlined above.

Alternatively, it is possible that the tree-level gaugino masses vanish, in which case eq. (2.1) (which holds trivially) is irrelevant. In this case, the gaugino mass parameters arise at one-loop. In particular, a model-independent contribution to the gaugino mass is present whose origin can be traced to the super-conformal (super-Weyl) anomaly, which is common to all supergravity models $[55,56]$. This contribution is dominant in models of anomaly-mediated supersymmetry breaking (AMSB). Eq. (2.1) is then replaced (in the one-loop approximation) by

$$
M_{i} \simeq \frac{b_{i} g_{i}^{2}}{16 \pi^{2}} m_{3 / 2}
$$

where $m_{3 / 2}$ is the gravitino mass (typically assumed to be on the order of $1 \mathrm{TeV}$ ), and the $b_{i}$ are the coefficients of the MSSM gauge beta-functions corresponding to the corresponding $\mathrm{U}(1)_{Y}, \mathrm{SU}(2)_{L}$, and $\mathrm{SU}(3)$ gauge groups: $\left(b_{1}, b_{2}, b_{3}\right)=\left(\frac{33}{5}, 1,-3\right)$. Eq. (2.2) yields $M_{1} \simeq$ $2.8 M_{2}$, which implies (under the assumption that $|\mu|$ is somewhat larger than $M_{2}$ ) that the lightest chargino pair and neutralino comprise a nearly mass-degenerate triplet of winos over most of the MSSM parameter space. Typically, the corresponding neutralino is the LSP, whereas the wino is the next-to-lightest supersymmetric particle (NLSP). ${ }^{5}$

The squarks that are produced in figure 1 are primarily first-generation squarks, either $\widetilde{q}_{L}$ or $\widetilde{q}_{R}$ (as the mixing between these states is negligible). In the case where the LSP is dominantly bino-like, then the branching ratio $\operatorname{BR}\left(\widetilde{q}_{R} \rightarrow q \widetilde{\chi}_{1}^{0}\right) \simeq 100 \%$. In contrast, the dominant channel for $\widetilde{q}_{L}$ decay is into the heavier wino-like neutralino which subsequently decays into the LSP. Thus, the production of $\widetilde{q}_{R}$ in association with the LSP is more likely to produce a monojet. In the case where the LSP is dominantly wino-like, the reverse is

\footnotetext{
${ }^{5}$ We ignore the gravitino, which could be lighter than the neutralino. Nevertheless, in almost all cases, the neutralino behaves as if it is the LSP. That is, the neutralino will be sufficiently long-lived so that it will always escape the collider detector before it decays.
} 
true, and the production of $\widetilde{q}_{L}$ in association with the LSP is more likely to produce a monojet.

In this paper we shall examine several scenarios. The first scenario considered will be an optimistic mSUGRA point, which will illustrate the effect of the SUSY backgrounds, making a coupling extraction from LHC data difficult. Next, we shall assume a wino dominated neutralino, where signal cross-sections are higher and easier to measure over the background. In this case, the coupling extraction from LHC data is somewhat easier. If the lightest neutralino is light and found to be wino dominated, then it does not constitute a significant portion of a thermal dark matter relic, since winos annihilate too efficiently in the early universe $[57,58]$.

\section{$3 \quad$ Testing the supersymmetric coupling relations with monojets}

\subsection{The signal process}

We define our parton-level signal processes in the following way:

- For a bino LSP: $g+u \rightarrow \widetilde{\chi}_{1}^{0}+\widetilde{u}_{R}, g+d \rightarrow \widetilde{\chi}_{1}^{0}+\widetilde{d}_{R}$ as displayed in figure 1 followed by $\widetilde{u}_{R} \rightarrow u \widetilde{\chi}_{1}^{0}$ or $\widetilde{d}_{R} \rightarrow d \widetilde{\chi}_{1}^{0}$. Here, $\lambda=g^{\prime}$, the $\mathrm{U}(1)_{Y}$ gauge coupling of the SM. The charge-conjugated processes are also included.

- For a wino LSP: $g+u \rightarrow \widetilde{\chi}_{1}^{0}+\widetilde{u}_{L}, g+d \rightarrow \widetilde{\chi}_{1}^{0}+\widetilde{d}_{L}, g+u \rightarrow \widetilde{\chi}_{1}^{+}+\widetilde{d}_{L}, g+d \rightarrow \widetilde{\chi}_{1}^{-}+\widetilde{u}_{L}$, where $\widetilde{u}_{L} \rightarrow u \widetilde{\chi}_{1}^{0} / d \widetilde{\chi}_{1}^{+}, \widetilde{d}_{L} \rightarrow d \widetilde{\chi}_{1}^{0} / u \widetilde{\chi}_{1}^{-}$and $\widetilde{\chi}_{1}^{+} \rightarrow S+\widetilde{\chi}_{1}^{0}$, where $S$ is either QCD radiation too soft to be identified as a jet, or a lepton so soft that it passes the lepton veto. For wino LSPs, the mass splitting between $\widetilde{\chi}_{1}^{+}$and $\widetilde{\chi}_{1}^{0}$ is $\sim 200 \mathrm{MeV}$, so the dominant decay $\widetilde{\chi}_{1}^{+} \rightarrow \widetilde{\chi}_{1}^{0} \pi^{+}$typically results in a soft pion that is too soft to be measured by the LHC experiments. Both the $\widetilde{\chi}_{1}^{0}$ and $\tilde{\chi}_{1}^{+}$are wino-like and are related via a $\mathrm{SU}(2)_{L}$ transformation, so the production process amplitudes are constrained by the MSSM to be proportional to $\lambda$, equal to the $\mathrm{SU}(2)_{L}$ gauge coupling $g$. The charge-conjugated processes are also included.

In principle, processes involving heavier non-valence quarks like $g+c \rightarrow \widetilde{\chi}_{1}^{0}+\widetilde{c}$ also contribute to our signal and SUSY backgrounds. We have only included initial states with a $g, u, \bar{u}, d$ or $\bar{d}$, since processes with quarks beyond the first generation are negligible. Since we assume that $\widetilde{\chi}_{1}^{0}$ is stable, it leaves no direct trace in the detector, and so the signal consists of a jet recoiling against apparently missing transverse momentum of magnitude $\not p_{T}$. In general, $\not \not_{T}$ is measured to be different to the transverse momentum of the jet $p_{T}(j)$ because of measurement errors and also because of soft QCD radiation, which may not be included in the jet, but measured in the calorimeter nonetheless.

\subsection{Major backgrounds and basic cuts}

As we have seen in the last section, our signal consists of a hard jet recoiling against missing energy. Therefore, the major SM backgrounds are [30, 59]

- $Z(\rightarrow \nu \bar{\nu})+$ jet. $Z$ production in association with a hard jet, where the $Z$ decays into a pair of neutrinos. 
- $W(\rightarrow \tau \nu)+$ jet. $W$ plus jet production, where the $W$ decays into a tau and a neutrino, and the tau is either not detected, or lost in the jet.

- $W(\rightarrow e / \mu \nu)+$ jet. $W$ plus jet production, where the $W$ decays into an electron or a muon and a neutrino and the electron/muon is undetected or lost inside the jet.

- QCD jet production together with mismeasurement of the energy deposited in the detector. One could produce di-jets, for instance, and one of the jets could be lost in the detector (or its energy mismeasured so that it fluctuate below the transverse momentum required to identify the jet).

In principle, di-vector boson production $V V^{\prime}$, with $V, V^{\prime}=W, Z$ is an additional source for the SM backgrounds if one vector decays into a neutrino and the other into jets. However, the cross section is much smaller than $Z / W+$ jet and $V V^{\prime}$ production can be safely neglected in our analysis [32].

In order to reject most of the $W+$ jet background, we employ a lepton veto. We veto events with an isolated electron or muon with $p_{T}>5 \mathrm{GeV}$ and with $|\eta|<2.5$. The isolation criterion demands less than $10 \mathrm{GeV}$ of additional energy in a cone of radius $\Delta R=\sqrt{\Delta \phi^{2}+\Delta \eta^{2}}=0.2$ around the lepton momentum, where $\phi$ and $\eta$ are the azimuthal angle and pseudorapidity, respectively.

After the lepton veto, $Z(\rightarrow \nu \bar{\nu})+$ jet remains the most important irreducible background. Fortunately, this background can be directly derived from data itself by measuring $Z\left(\rightarrow e^{+} e^{-} / \mu^{+} \mu^{-}\right)+$jet and relating both processes with the help of the measured $Z$ branching ratios. Systematic uncertainties are in this case significantly reduced [60]. However, this comes with the cost of higher statistical uncertainties, because the $Z(\rightarrow \nu \bar{\nu})+$ jet cross section is roughly three times larger than the $Z\left(\rightarrow e^{+} e^{-} / \mu^{+} \mu^{-}\right)+$jet cross section. $\gamma+$ jet cross-sections at high $p_{T}$ could be used in the future to estimate the $Z(\rightarrow \nu \bar{\nu})+$ jet cross section with better statistics.

We will use two statistical estimators; cf. refs. [30, 59]. The first optimistic estimator takes only the statistical fluctuations from $Z(\rightarrow \nu \bar{\nu})+$ jet into account. In this case the significance is given by $S / \sqrt{B}$ with $S(B)$ the number of expected signal (SM background) events. This case would apply when the background Monte-Carlo is so well tuned and tested with LHC data that its output may be fully trusted. We also employ a conservative estimator, where the statistical fluctuations are dominated by the $Z\left(\rightarrow e^{+} e^{-} / \mu^{+} \mu^{-}\right)+$jet calibration sample. According to refs. [30, 59], this case corresponds to a significance of $S / \sqrt{7 B}{ }^{6}$ The conservative estimate would be used in the case that one does not trust at all the Monte-Carlo background calculation, and instead measures the $Z+$ jet background from LHC data. We expect the true (i.e. measurable) significance to lie between our two estimators after the Monte Carlo estimates [60] have been properly tuned to LHC data, including $W+$ jet $/ \gamma+$ jet. The modeling of systematic error on the measurements of SM backgrounds is an experimental question, and as such is beyond the scope of this paper.

\footnotetext{
${ }^{6}$ In refs. $[30,59]$ the transverse momentum of the monojet was required to lie above $1 \mathrm{TeV}$. In contrast, we will use less hard cuts for which the $Z\left(\rightarrow e^{+} e^{-} / \mu^{+} \mu^{-}\right)+$jet acceptance is slightly better; see ref. [59]. However, we will always use $S / \sqrt{7 B}$ as a conservative estimator.
} 
After cuts, once the large $Z(\rightarrow \nu \bar{\nu})+$ jet background is subtracted, we will still need to subtract the $W+$ jet background, which is larger than our signal. This can be measured in the case where the lepton from the $W$ is visible, by extrapolating into the region where the lepton is invisible, either because it is lost in the jet (in which case one could extrapolate in $\Delta R$ between jet and lepton), or because the lepton is missed.

An estimate of the QCD background can be found by full detector simulation [60]. The quantity of interest for estimating these backgrounds is the jet energy response function (JERF) $R$, equal to the ratio of the measured jet energy to the true one. We fit the JERF to the full detector simulation results in [60] in the same spirit as ref. [61]. In our analysis, $R$ is used to scale all of the components of the jet four-momentum. $R$ is well fit by a probability distribution function

$$
p(R)=\frac{0.99}{\sqrt{2 \pi \sigma^{2}}} e^{\frac{(1-R)^{2}}{2 \sigma^{2}}}+0.01 A e^{7.32 R} \Theta(0.9-R),
$$

where $\Theta$ is the Heaviside step function, $A$ is an unimportant normalization constant $(\approx 0.01)$, and

$$
\sigma=\frac{0.6}{\sqrt{E / \mathrm{GeV}}} \oplus 0.03
$$

is the resolution of the Gaussian part of the JERF, and $\oplus$ denotes the fact that the two terms are added in quadrature. The second term in $p(R)$ reflects the $1 \%$ probability that a significant portion of the jet will go unmeasured due to cracks in the detector and other effects. This exponential tail is responsible for the QCD background to our SUSY monojet signal, and in order to increase statistics on the sample, we impose that either the hardest or second hardest jet in the sample be in the Gaussian tail, taking into account the additional factor of 100 needed to calculate the cross-section after cuts. Other jets are all drawn from $p(R)$. QCD backgrounds to monojets will be extrapolated from data, for example from di-jet, $\gamma+$ jet and "Mercedes" type 3 -jet events [60], although we note that these techniques may not address more conspiratorial backgrounds, which could require the use of tracking information. QCD backgrounds will then be subtracted from event samples, leaving statistical fluctuations only once the systematics have been dealt with. The JERF was only applied to QCD backgrounds, not to the other samples, since it should only have a small effect upon them. We note that Herwig++ includes $b$ backgrounds within this QCD sample, so the case of the production of $b \bar{b}$, where one of the bottom quarks' momenta primarily goes into a neutrino resulting in a monojet signature, is included in our estimate.

A further background source is supersymmetry itself. For example, squark pair production and the subsequent decay of the squarks into the $\widetilde{\chi}_{1}^{0}$ LSP and a quark is a possible SUSY background. If the two jets from the squark decays overlap they can appear as a single monojet. Initial-state radiation on $\widetilde{\chi}_{1}^{0} \widetilde{\chi}_{1}^{0}$ production also provides a SUSY background, although this is usually rather small. Note that the background of squark pair production followed by the decay into two jets plus two $\widetilde{\chi}_{1}^{0}$, where one of the jets is in the tail of the JERF, is not in our SUSY background sample. We expect this background to be smaller than the QCD background, which is already small. 


\begin{tabular}{|c|c|c|}
\hline sample & simulated events & comments \\
\hline$Z(\rightarrow \nu \bar{\nu})+$ jet & 2140000 & $p_{T}($ jet $)>70 \mathrm{GeV}$ \\
$W(\rightarrow e \nu)+$ jet & 2960000 & $p_{T}($ jet $)>70 \mathrm{GeV}$ \\
$W(\rightarrow \mu \nu)+$ jet & 2960000 & $p_{T}($ jet $)>70 \mathrm{GeV}$ \\
$W(\rightarrow \tau \nu)+$ jet & 2960000 & $p_{T}($ jet $)>70 \mathrm{GeV}$ \\
QCD & 18650000 & $p_{T}($ jet $)>30 \mathrm{GeV}$ \\
\hline mSUGRA & 3280000 & Included 10300 signal events. \\
mAMSB & 2940000 & Included 46700 signal events. \\
others & 100000 & Number of signal events varies. \\
\hline
\end{tabular}

Table 1. Monte Carlo samples of SM backgrounds and SUSY events used in our analysis. All simulated events were generated with Herwig++2.4.2 for $p p$ collisions at a center-of-mass energy of $14 \mathrm{TeV}$. The $Z+$ jet and $W+$ jet samples correspond to an integrated luminosity of $5 \mathrm{fb}^{-1}$. The QCD sample corresponds to $30 \mathrm{nb}^{-1}$, the mSUGRA sample corresponds to $20 \mathrm{fb}^{-1}$ and the mAMSB sample corresponds to $100 \mathrm{fb}^{-1}$. The cut on $p_{T}$ (jet) is performed at the parton level. The mSUGRA [mAMSB] sample corresponds to sparticle pair production assuming the benchmark scenario eq. (4.1) [eq. (5.1)]; the number of SUSY events that correspond to our signal monojet events is indicated in the third column of the table for these two rows. The scenarios denoted by others are those of the parameter scan of section 5.4; the corresponding integrated luminosities have been adjusted for each scan point such that 100000 events for each point are generated.

In the case of a bino-like LSP, $\widetilde{\chi}_{2}^{0}+\widetilde{q}_{L}$ production followed by $\widetilde{\chi}_{2}^{0} \rightarrow \widetilde{\chi}_{1}^{0}+\nu \bar{\nu}$ and $\widetilde{q}_{L} \rightarrow \widetilde{\chi}_{1}^{0}+q$ would also produce monojet events. The cross-section for $\widetilde{\chi}_{2}^{0}+\widetilde{q}_{L}$ is enhanced by $K g^{2} / g^{\prime 2} \simeq 2$ over $\widetilde{\chi}_{1}^{0}+\widetilde{q}_{R}$ production (where the kinematical factor $K$ provides a suppression due to the heavier $\widetilde{\chi}_{2}^{0}$ mass). However, due to the branching ratio factors for the final state decays, the number of $\widetilde{\chi}_{2}^{0}+\widetilde{q}_{L}$ events that are observed as monojets is significantly smaller than our signal and can be neglected.

In the case of a (not too heavy; see section 5.4) wino-like LSP, the dominant SUSY background is wino pair production plus a jet from initial state radiation. The cross section is quite large because two winos, $\widetilde{W} \widetilde{W}$, can be produced via a Drell-Yan like process [62, 63], i.e. $P P \rightarrow \gamma^{*} / Z^{*} \rightarrow \widetilde{W} \widetilde{W}$. Here, $\gamma^{*}\left(Z^{*}\right)$ denotes a virtual $\gamma(Z)$. In the wino-LSP scenario, both $\widetilde{W}$ means either $\widetilde{\chi}_{1}^{ \pm}$or $\widetilde{\chi}_{1}^{0}$, both of which are approximately winos. Because the $\widetilde{\chi}_{1}^{ \pm}$ is quasi-degenerate in mass with the $\widetilde{\chi}_{1}^{0}$, it decays into a soft pion and the $\widetilde{\chi}_{1}^{0}$. The pion is typically too soft to be detected $[57,58]$ unless special analysis techniques are used [64] and so the $\tilde{\chi}_{1}^{ \pm}$is effectively invisible to the detector. The additional jet is then produced via initial state radiation.

We have employed Herwig++2.4.2 $[65-67]^{7}$ to simulate the signal and the backgrounds at tree level in $p p$ collisions at a center-of-mass energy of $14 \mathrm{TeV}$. An overview of the simulated signal and background samples is given in table 1. We have included the pair production and the two and three-body decays of all SUSY particles. All events from this sample that yield the correct monojet topology that are not our signal process are classified as SUSY background. The $Z+$ jet/ $W+$ jet backgrounds are also obtained with Herwig++.

\footnotetext{
${ }^{7}$ We have used a modified version of Herwig++2.4.2 which is also able to deal with negative (Majorana) gluino masses; see the following link for details [68].
} 


\begin{tabular}{|cc|cc|}
\hline sparticle & mass $[\mathrm{GeV}]$ & sparticle & mass $[\mathrm{GeV}]$ \\
\hline$\widetilde{\chi}_{1}^{0}$ & 70.2 & $\widetilde{\chi}_{4}^{0}$ & 365 \\
$\widetilde{\chi}_{1}^{+}$ & 132 & $\widetilde{\chi}_{2}^{+}$ & 370 \\
$\widetilde{\chi}_{2}^{0}$ & 133 & $\widetilde{b}_{1}$ & 378 \\
$\widetilde{\tau}_{1}$ & 189 & $\widetilde{b}_{2}$ & 443 \\
$\widetilde{t}_{1}$ & 226 & $\widetilde{u}_{R} / \widetilde{c}_{R}$ & 454 \\
$\widetilde{\nu}_{\tau}$ & 230 & $\widetilde{d}_{R} / \widetilde{s}_{R}$ & 455 \\
$\widetilde{e}_{R} / \widetilde{\mu}_{R}$ & 234 & $\widetilde{g}$ & 456 \\
$\widetilde{\nu}_{e} / \widetilde{\nu}_{\mu}$ & 242 & $\widetilde{u}_{L} / \widetilde{c}_{L}$ & 463 \\
$\widetilde{e}_{L} / \widetilde{\mu}_{L}$ & 255 & $\widetilde{d}_{L} / \widetilde{s}_{L}$ & 470 \\
$\widetilde{\tau}_{2}$ & 259 & $\widetilde{t}_{2}$ & 477 \\
$\widetilde{\chi}_{3}^{0}$ & 359 & & \\
\hline
\end{tabular}

Table 2. Sparticle mass spectrum of the mSUGRA benchmark scenario given by eq. (4.1). The sparticles are ordered by their mass.

The Herwig++ output was analyzed using HepMC-2.04.02 [69] and ROOT [70, 71]. Jets were reconstructed with the help of fastjet-2.4.1 [72]. We employed the anti- $k_{t}$ jet algorithm with $R=0.7$ [73]. Only jets with $p_{T}>30 \mathrm{GeV}$ are used in our analysis in order to be less sensitive to underlying event modeling. In table 1 , we list the total number of simulated events for each sample. In the bottom half of the table, the total number of SUSY pair production events is listed first, with the number in the subset corresponding to our signal events listed under "comments".

\section{Measurement of the $\widetilde{\chi}_{1}^{0} \widetilde{q} q$ coupling for a bino LSP}

In this section we will show that it is possible to estimate the $\widetilde{\chi}_{1}^{0} \widetilde{q}_{R} q$ coupling for the case of a bino-like $\widetilde{\chi}_{1}^{0}$ LSP from data. We choose an mSUGRA benchmark scenario for investigation.

\subsection{Benchmark scenario}

Our benchmark scenario is a light mSUGRA scenario [74-80] with a bino-like $\widetilde{\chi}_{1}^{0}$ LSP. It is described by the parameters

$$
M_{0}=220 \mathrm{GeV}, M_{1 / 2}=180 \mathrm{GeV}, A_{0}=-500 \mathrm{GeV}, \tan \beta=20, \operatorname{sgn}(\mu)=+1 .
$$

For this scenario, the $\widetilde{\chi}_{1}^{0} \widetilde{q}_{R} q$ coupling is given by $\lambda=0.99 g^{\prime}$, where the deviation from $\lambda=g^{\prime}$ is due to the small admixture of wino and higgsino eigenstates in the LSP. All spectra are calculated with SOFTSUSY3.0.13 [81] and fed into the event generator via the SUSY Les Houches Accord [82]. The sparticle masses are given in table 2.

This scenario has a relatively light SUSY mass spectrum and a total signal cross-section of $\sigma\left(p p \rightarrow \widetilde{q}_{R} \widetilde{\chi}_{1}^{0}\right)=520 \mathrm{fb}$. For spectra a little heavier, the monojet cross section is too small and could not be seen above the SM backgrounds. Note that the mSUGRA scenario, eq. (4.1), lies at the edge of the region excluded by the Tevatron, but is still allowed [83, 84]. 


\begin{tabular}{|l|ccc|c|}
\hline cut & all SM & SUSY bkg. & signal & $S / \sqrt{B}$ \\
\hline trigger & $1.14 \times 10^{8}$ & $2.91 \times 10^{7}$ & 130000 & - \\
lepton veto & $7.57 \times 10^{7}$ & $1.76 \times 10^{7}$ & 130000 & - \\
number $($ jets $)=1$ & $3.35 \times 10^{7}$ & 55900 & 35100 & $6.1(2.3)$ \\
$\not_{T}>180 \mathrm{GeV}$ & $3.28 \times 10^{6}$ & 32300 & 22300 & $12(4.7)$ \\
$m($ jet $)<70 \mathrm{GeV}$ & $3.00 \times 10^{6}$ & 12100 & 20100 & $12(4.4)$ \\
\hline tau veto & $2.75 \times 10^{6}$ & 9950 & 20000 & $12(4.6)$ \\
$b$-jet veto & $2.66 \times 10^{6}$ & 9290 & 20000 & $12(4.6)$ \\
\hline
\end{tabular}

Table 3. Cut flow for the mSUGRA benchmark scenario in eq. (4.1). We present the cuts in the first column and the number of SM, SUSY background and signal events in the second, third and fourth column, respectively. We also show in the fifth column the resulting significance for the monojet signal, i.e. $B$ corresponds to the number of SM background events and $S$ is the number of signal events. The significances in brackets are our conservative estimate, i.e. $S / \sqrt{7 B}$. We have assumed an integrated luminosity of $300 \mathrm{fb}^{-1}$ at $\sqrt{s}=14 \mathrm{TeV}$. Note that in addition to the $p_{T}$ cut, the same cut has been applied on the jet- $p_{T}$.

\subsection{Event numbers and cuts}

In this section, we will develop a set of cuts that allow a measurement of the $\widetilde{\chi}_{1}^{0} \widetilde{q}_{R} q$ coupling assuming our mSUGRA benchmark scenario, eq. (4.1). On one hand, the number of signal events needs to significantly exceed the statistical fluctuations of the SM backgrounds. On the other hand, we desire a good signal to SUSY background ratio if we want to measure $\lambda$ to a high precision.

In table 3, we display the total number of SM background events (second column), the number of SUSY background events (third column) and the number of signal events (fourth column) for different sets of cuts (first column). We also show in the fifth column the resulting significance for the monojet signal, i.e. $B$ corresponds to the number of SM background events and $S$ is the number of signal events. Note that $B$ refers to the SM background only, but the SM contribution completely dominates the background. We assumed an integrated luminosity of $300 \mathrm{fb}^{-1}$ at $\sqrt{s}=14 \mathrm{TeV}$. We cannot give meaningful numbers for the significances in the first two rows because SM backgrounds additional to those of section 3.2 would need to be included.

As a first cut (denoted by trigger), we demand at least one jet with transverse momentum, $p_{T}$ (jet), larger than $100 \mathrm{GeV}$. In addition, the amount of missing transverse momentum, $\not{ }_{T}$, must also exceed $100 \mathrm{GeV}$. These cuts correspond to the planned one-jet plus missing energy trigger used by the ATLAS collaboration [85] for the $14 \mathrm{TeV}$ run. Therefore, all events in the first row of table 3 will be recorded. Note that CMS plans to use harder cuts [86]. We can see in table 3 that two orders of magnitude more SUSY background events will pass the trigger than signal events.

The SUSY background is reduced by roughly a factor of two after we apply (in addition to the trigger cut) a veto on isolated electrons and muons; see section 3.2. At the same time, the number of signal events is nearly unaffected. The SM backgrounds are also reduced by the lepton veto, because most of the $W+$ jet background events will not pass this cut. 


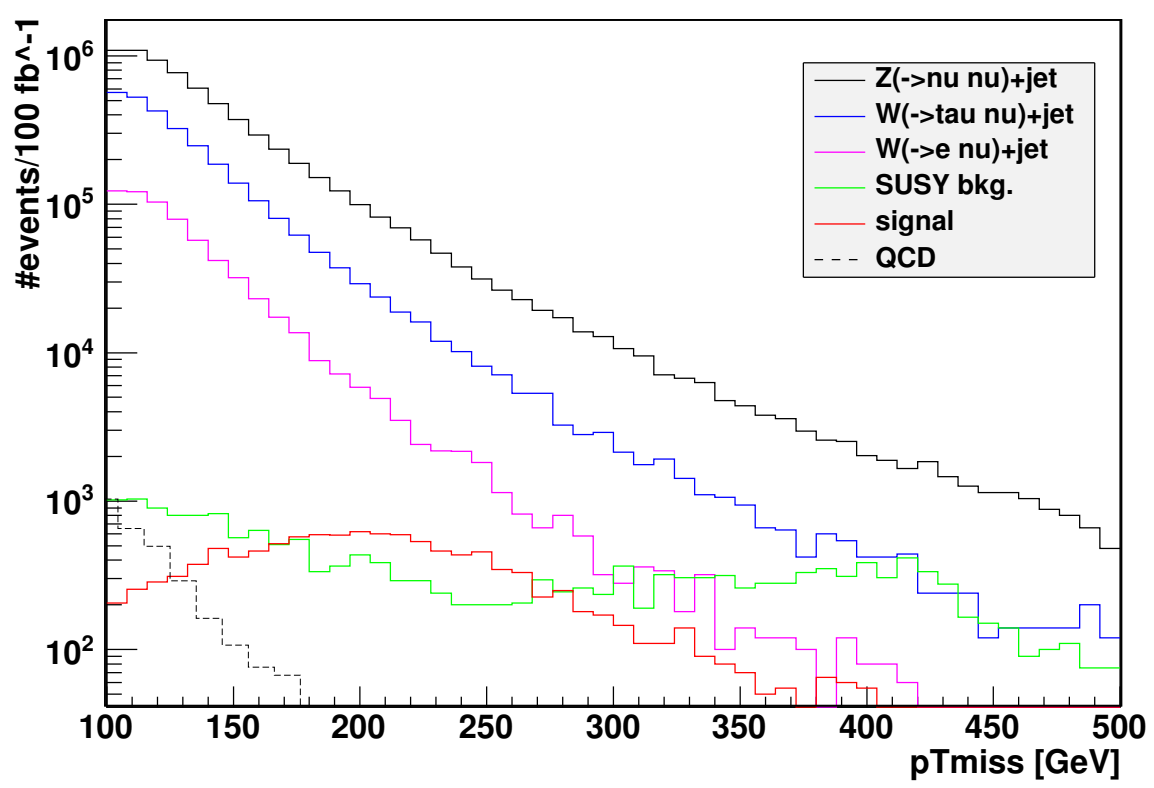

Figure 3. Missing transverse momentum distribution for the $Z(\rightarrow \nu \bar{\nu})+$ jet (black histogram), $W(\rightarrow \tau \nu)+$ jet (blue histogram), $W(\rightarrow e \nu)+$ jet (magenta histogram), the SUSY background (green histogram), the QCD background (black dashed histogram) and the signal process (red histogram). The first three cuts of table 3 have been applied. The number of events correspond to an integrated luminosity of $100 \mathrm{fb}^{-1}$ at $\sqrt{s}=14 \mathrm{TeV}$. We assume the mSUGRA scenario of eq. (4.1).

However, the $Z(\rightarrow \nu \bar{\nu})+$ jet background stays nearly the same.

To reduce the SUSY background further, we also apply a veto on a second jet if its $p_{T}$ is larger than $30 \mathrm{GeV}$ and $|\eta|<5$. This corresponds to a veto on a second jet, because we only count jets above $30 \mathrm{GeV}$, cf. section 3.2. The number of SUSY background events now has the same order of magnitude as the signal, namely $\mathcal{O}\left(10^{4}\right)$ events. Although the signal possesses no second jet at parton level, we might produce one due to initial and final state radiation, as is borne out by the third row of table 3 . In particular, a veto on a second jet also reduces the number of signal events by roughly a factor of four.

At this stage we are able to give some reliable numbers for signal significances, assuming that backgrounds can be reliably constrained and subtracted. The numbers in brackets correspond to our conservative estimate as described in section 3.2, i.e. we assume that the dominant $Z(\rightarrow \nu \bar{\nu})+$ jet background is estimated purely from a measurement of $Z(\rightarrow$ $\left.e^{+} e^{-} / \mu^{+} \mu^{-}\right)+$jet. We observe an optimistic (conservative) significance for the monojet signal of $6.1(2.3)$.

In order to increase the signal to SM background ratio we make use of the $\not_{T}$ and jet- $p_{T}$ distribution of our monojet signal. They possess a Jacobian peak, where the position depends on the $\widetilde{q}_{R}$ and $\widetilde{\chi}_{1}^{0}$ masses (see appendix A). We present the $\not_{T}$ distributions in figure 3 . The Jacobian peak of our monojet signal (red histogram) lies around $180 \mathrm{GeV}$. At the same time the distributions of the SM backgrounds (black, blue, magenta and dashed black histograms) fall off exponentially. An additional lower bound on $\not_{T}$ and the monojet 


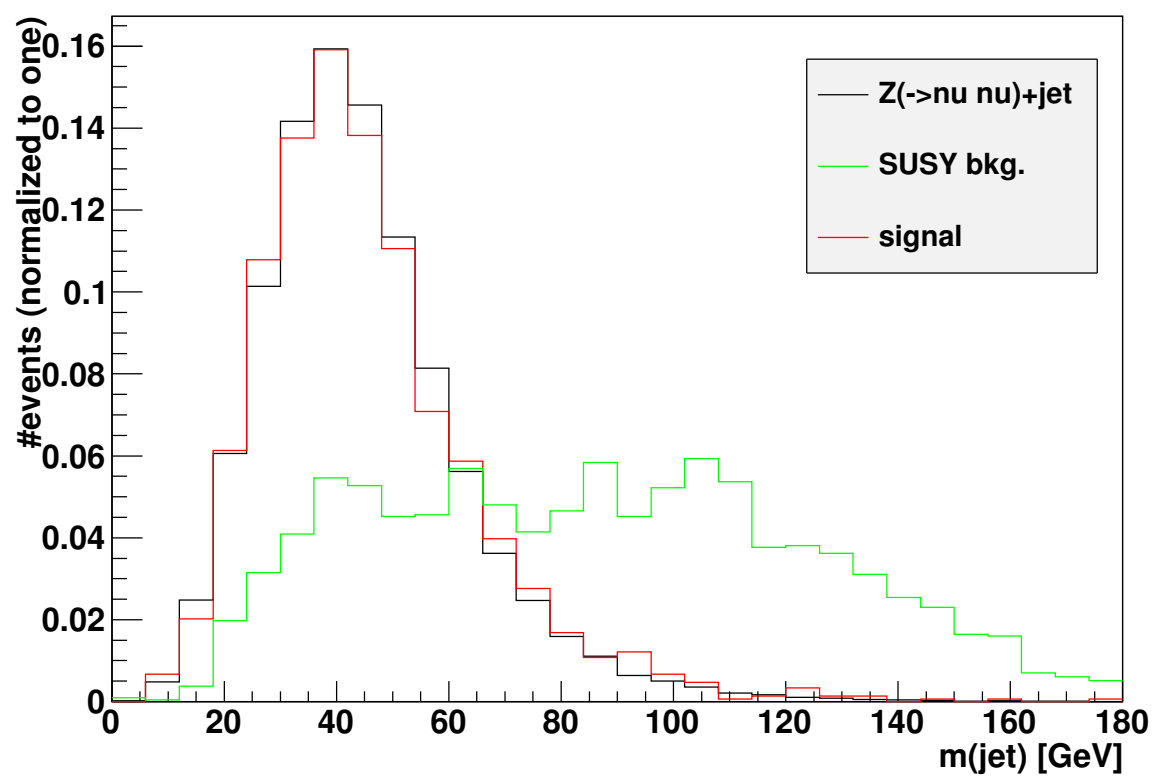

Figure 4. Invariant mass distribution of the hardest jet for the $Z(\rightarrow \nu \bar{\nu})+$ jet background (black histogram), the SUSY background (green histogram) and the signal process (red histogram). The first four cuts of table 3 have been applied. The distributions are normalized to one. For the signal, we assume the mSUGRA scenario of eq. (4.1). The $W+$ jet and QCD backgrounds have a distribution almost indistinguishable from that of $Z(\rightarrow \nu \bar{\nu})+$ jet.

$p_{T}$ of $180 \mathrm{GeV}$, raises the optimistic (conservative) signal versus SM background significance to 12 (4.7). The required level of cancellation is high: around 1 in 100 for a high $\not{ }_{T}$ cut. This means that the experimental systematic on the dominant background also needs to be at the level of $\lesssim 1 \%$, a challenging (but not obviously impossible) proposition. The experimental systematic on the subdominant $W+$ jet background subtraction would need to be at the level of a few percent.

Our goal is to reconstruct the $\widetilde{\chi}_{1}^{0} \widetilde{q}_{R} q$ coupling as precisely as possible. In order to accomplish this goal, we need to reduce the SUSY background further while leaving the number of signal events unchanged (in order to have a visible signal). For this purpose, we have examined the invariant mass of the hardest jet, $m$ (jet), which is presented in figure 4 for the signal (red histogram), the SUSY background (green histogram) and the $Z+$ jet background (black histogram). Each histogram is separately normalized so that the total number of events is one. The first four cuts of table 3 are applied. Note that the shape of $m$ (jet) for $W+$ jet and QCD backgrounds follows those of $Z+$ jet.

We first observe that the distribution of the $Z+$ jet background looks very similar to the signal distribution. This is expected; the $m$ (jet) distribution for these two cases is what is predicted by an approximately massless initial parton (which ideally would peak at zero mass) when one applies lower $p_{T}$ (jet) cuts, cutting out the very low mass region. Therefore, a cut on $m$ (jet) cannot increase the signal significance of the signal over SM background. However, the SUSY background distribution has a very different shape. It is relatively flat compared to the signal and has its maximum at a larger $m$ (jet) value. The (on average) larger jet invariant mass of the SUSY background stems mainly from events, 


\begin{tabular}{|l|cc|}
\hline error & $\Delta \sigma_{\text {mono }} / \sigma_{\text {mono }}$ & $\Delta \lambda / \lambda$ \\
\hline luminosity & $3.0 \%$ & $1.5 \%$ \\
PDF uncertainty & $17 \%$ & $8.3 \%$ \\
NLO corrections & $16 \%$ & $8.0 \%$ \\
sparticle mass $\Delta \tilde{m}=10 \mathrm{GeV}$ & $11 \%$ & $5.6 \%$ \\
statistics (optimistic) & $8.6 \%$ & $4.3 \%$ \\
statistics (conservative) & $23 \%$ & $11 \%$ \\
\hline total (optimistic) & $27 \%$ & $14 \%$ \\
total (conservative) & $35 \%$ & $17 \%$ \\
\hline
\end{tabular}

Table 4. Relative errors for the signal monojet cross section (second column) and the $\widetilde{\chi}_{1}^{0} \widetilde{q}_{R} q$ coupling $\lambda$ (third column) from different sources (first column). The numbers are for the mSUGRA benchmark scenario, eq. (4.1).

where a pair of squarks is produced and where the jets from squark decays go roughly in the same direction and are identified as only one jet. Relative to the cuts listed in table 3 , imposing the requirement that $m$ (jet) is less than $70 \mathrm{GeV}$ reduces the SUSY background by roughly a factor of three and leaves the number of signal events nearly unchanged. ${ }^{8}$ Note that the cut on $m$ (jet) mostly suppresses the high- $\not{ }_{T}$ SUSY background events in figure 3 , i.e. $\not \not_{T} \gtrsim 300 \mathrm{GeV}$. In the next section, we will employ these cuts, i.e. the first five cuts in table 3 , for the coupling reconstruction.

Finally, in the last two rows of table 3 we show the effects of a tau-lepton veto and a subsequent $b$-jet veto. In both cases we assumed an identification (ID) efficiency of $100 \%$. We regard a tau as identified if it decays hadronically and has $p_{T}>15 \mathrm{GeV}$ and $|\eta|<2.5$. We observe that neither veto helps much, and so their effect would be even more reduced if realistic ID efficiencies were assumed. We see that the SUSY background would be reduced by $18 \%$. However, the ID efficiencies are doubtless too optimistic and in reality the suppression of the SUSY background would lie below $11 \%[60,87]$. Thus, we will not employ the tau and $b$-jet vetoes here.

\subsection{Coupling reconstruction}

We now show to which precision the $\widetilde{\chi}_{1}^{0} \widetilde{q}_{R} q$ coupling $\lambda$ of the mSUGRA benchmark scenario of section 4.1 can be reconstructed with an integrated luminosity of $300 \mathrm{fb}^{-1}$ at $\sqrt{s}=$ $14 \mathrm{TeV}$. We employ the cuts developed in section 4.2 (without the tau and $b$-jet veto). Since the signal monojet cross section is proportional to $\lambda^{2}$, the relative error on $\lambda$ will be roughly one half of the relative error on the monojet production cross section. Note that we reconstruct $\lambda$ under the assumption that the SUSY backgrounds come from our mSUGRA point.

In table 4, we show the statistical and the most important systematic errors for a measurement of the signal cross section and $\widetilde{\chi}_{1}^{0} \widetilde{q}_{R} q$ coupling $\lambda$. The total error is obtained

\footnotetext{
${ }^{8}$ For the mSUGRA benchmark point, the number of surviving monojet events after the cut on $m$ (jet) arising from $\widetilde{\chi}_{2}^{0}+\widetilde{q}_{L}$ production (which is included in the SUSY background in figure 4) is about $5 \%$ of our signal events.
} 
by combining all errors in quadrature. The errors were estimated as follows:

- In order to determine the monojet cross section from the observed number of events, one needs to know the total number of collisions, i.e. the integrated luminosity. According to ref. [88-90], the integrated luminosity at LHC will be measured to a precision of $3 \%$ or better.

- To translate the hadronic cross section to a parton-level cross section, one needs to know the parton distribution functions (PDFs) accurately. We estimated the error from the PDFs by comparing the hadronic cross sections with different PDF releases; namely CTEQ61, CTEQ61l, CTEQ6m [91], MSTW2008lo, MSTW2008nlo, MSTW2008nnlo [92], GJR08 [93, 94] and a cubic interpolation of MRST LO** [95] (default Herwig++ PDF). We found the largest variation between MRST LO** and MSTW2008lo. It corresponds to a variation of $\pm 17 \%$ around the central value of the total hadronic signal cross section. We expect the PDF errors to be reduced significantly after the input to PDFs of LHC data, but for now we use the current value of the error.

- Further uncertainties arise from the unknown next-to-leading order (NLO) corrections, which are not yet fully implemented [96]. The corresponding uncertainty is estimated by varying the renormalization scale $Q$ between $\frac{1}{4}\left(m_{\tilde{q}_{R}}+m_{\tilde{\chi}_{1}^{0}}\right)<Q<$ $m_{\tilde{q}_{R}}+m_{\tilde{\chi}_{1}^{0}}$ (where the average squark mass of the right-handed first and second generation squarks is denoted by $m_{\tilde{q}_{R}}$ ) leading to an error of $+16 \% /-13 \%$ for the total LO cross section. In table 7 , we use $16 \%$ in order to be rather conservative, with the knowledge that the NLO calculation would be ready and significantly decrease (possibly by a factor of a few) this uncertainty. The LO cross section was derived with Prospino2.1 [97].

- The relatively light SUSY spectrum in table 2 leads to copious production of SUSY particles at the LHC [98]. In this case, we expect that the $\widetilde{\chi}_{1}^{0}$ and squark masses can be reconstructed to a precision of at least $10 \mathrm{GeV}$ [4]. Varying the squark and $\widetilde{\chi}_{1}^{0}$ masses by $\pm 10 \mathrm{GeV}$ introduces an error of $\pm 11 \%$ around the central value of the total monojet cross section.

- We always get an error due to statistical fluctuations of the SM backgrounds. In table 7, we present two statistical estimates for this error as described in section 3.2. The optimistic (conservative) estimator is $\sqrt{B}(\sqrt{7 B})$, with $B$ the number of SM background events.

We have neglected in table 4 errors from the SUSY background, because an precise estimate of its systematic uncertainties lies beyond the scope of this publication. However, we expect them to be small, because it is in principle possible to extrapolate them from data. As we observe in figure 4, the invariant mass distribution of the monojet is dominated by the SUSY background for $m$ (jet) $>70 \mathrm{GeV}$ providing a control sample for a fit of the SUSY background. Further model assumptions will also reduce the systematic uncertainties. 
We can see in table 4 that the biggest uncertainties are: the PDF uncertainty, NLO corrections and from the SM statistics (conservative estimate). However, one can hope that the uncertainty of the PDFs will rapidly decrease after LHC has taken and analyzed some first data. Furthermore, the calculations of higher order corrections might improve in the future. Unfortunately, an integrated luminosity of $300 \mathrm{fb}^{-1}$ corresponds already to the maximum expected LHC data. Some improvement might be possible by combining the data of both multi purpose detectors.

To conclude, a measurement of $\lambda$ via monojet production at the LHC is in principle possible for scenarios with a bino-like $\widetilde{\chi}_{1}^{0}$ LSP like mSUGRA. However, at least parts of the SUSY mass spectrum need to be relatively light, i.e. the right-handed up-squark and down-squark $\left(\widetilde{\chi}_{1}^{0}\right.$ LSP) mass should be $\lesssim 500 \mathrm{GeV}(\lesssim 100 \mathrm{GeV})$. A measurement of the $\widetilde{\chi}_{1}^{0} \widetilde{q}_{R} q$ coupling at the $10 \%$-level is feasible by the end of LHC running. The bino couplings to quark-squark pairs is proportional to the corresponding squark hypercharge $Y$, which is different for $\widetilde{d}_{R}$, and $\widetilde{u}_{R}$. Since production of both squarks contributes to our SUSY monojet signal, this fact must be taken into account when extracting a value of $\lambda$ from the data. ${ }^{9}$ For much larger masses, the monojet cross section is too small to allow its extraction.

The situation is much more promising for SUSY scenarios with a wino-like $\widetilde{\chi}_{1}^{0}$ LSP. The monojet cross section is now enhanced compared to the bino-LSP case due to larger gauge couplings and due to additional diagrams involving a charged wino.

\section{$5 \quad$ Reconstruction of the $\widetilde{\chi}_{1}^{0} \widetilde{q} q$ coupling for a wino LSP}

\subsection{Benchmark scenario}

The second benchmark scenario is a mAMSB scenario $[55-58,99,100]$ with a wino-like $\widetilde{\chi}_{1}^{0}$ LSP and with a wino-like $\widetilde{\chi}_{1}^{+}$NLSP that is nearly degenerate in mass with the $\widetilde{\chi}_{1}^{0}$ LSP. The scenario is described by the parameters

$$
M_{3 / 2}=33 \mathrm{TeV}, \quad M_{0}=200 \mathrm{GeV}, \quad \tan \beta=10, \quad \operatorname{sgn}(\mu)=+1 .
$$

The resulting sparticle masses at the electroweak scale are given in table 5 .

In this scenario, the total signal cross-section is $\sigma\left(p p \rightarrow \widetilde{q}_{L} \widetilde{\chi}_{1}^{0} / \widetilde{\chi}_{1}^{+}\right)=470 \mathrm{fb}$, and $\lambda=0.99 g$ due to the small bino/higgsino admixture. The lightest chargino, which will be included in our signal, couples with strength $0.98 g$ to $\widetilde{d}_{L} u$ because of a small higgsino admixture and with $1.00 \mathrm{~g}$ to $\widetilde{u}_{L} d$. The small differences between the $\widetilde{\chi}_{1}^{ \pm}$and $\widetilde{\chi}_{1}^{0}$ couplings are much smaller than our measurement errors, and shall be neglected in the following.

\subsection{Event numbers and cuts}

In this section we apply the same procedure as we did in section 4.2. We develop a set of cuts such that a clear signal over the SM backgrounds is visible. Furthermore, we want to

\footnotetext{
${ }^{9}$ We expect that the first generation squarks are roughly mass-degenerate. However, if there were a significant hierarchy between the up and down-type squark masses, then one would dominantly produce the lightest squark, and the extraction of $\lambda$ from data would be more straightforward.
} 


\begin{tabular}{|cc|cc|}
\hline sparticle & mass $[\mathrm{GeV}]$ & sparticle & mass $[\mathrm{GeV}]$ \\
\hline$\widetilde{\chi}_{1}^{0}$ & 106.5 & $\widetilde{\chi}_{4}^{0}$ & 593 \\
$\widetilde{\chi}_{1}^{+}$ & 106.7 & $\widetilde{\chi}_{2}^{+}$ & 594 \\
$\widetilde{\tau}_{1}$ & 113 & $\widetilde{b}_{1}$ & 634 \\
$\widetilde{\nu}_{\tau}$ & 135 & $\widetilde{t}_{2}$ & 688 \\
$\widetilde{\nu}_{e} / \widetilde{\nu}_{\mu}$ & 138 & $\widetilde{u}_{L} / \widetilde{c}_{L}$ & 722 \\
$\widetilde{e}_{R} / \widetilde{\mu}_{R}$ & 150 & $\widetilde{b}_{2}$ & 723 \\
$\widetilde{e}_{L} / \widetilde{\mu}_{L}$ & 159 & $\widetilde{d}_{L} / \widetilde{s}_{L}$ & 726 \\
$\widetilde{\tau}_{2}$ & 179 & $\widetilde{u}_{R} / \widetilde{c}_{R}$ & 726 \\
$\widetilde{\chi}_{2}^{0}$ & 298 & $\widetilde{d}_{R} / \widetilde{s}_{R}$ & 732 \\
$\widetilde{t}_{1}$ & 521 & $\widetilde{g}$ & 745 \\
$\widetilde{\chi}_{3}^{0}$ & 584 & & \\
\hline
\end{tabular}

Table 5. Supersymmetric particle masses for the mAMSB scenario defined in eq. (5.1). We show the masses of the wino-like $\widetilde{\chi}_{1}^{0}$ and $\widetilde{\chi}_{1}^{+}$up to a precision of four digits, because they are nearly degenerate in mass. The sparticles are ordered by mass.

\begin{tabular}{|l|ccc|c|}
\hline cut & all SM & SUSY bkg. & signal & $S / \sqrt{B}$ \\
\hline trigger & $3.81 \times 10^{7}$ & $1.04 \times 10^{6}$ & 44100 & - \\
lepton veto & $2.52 \times 10^{7}$ & 621000 & 43800 & - \\
$p_{T}($ jet 2$)<50 \mathrm{GeV}$ & $1.73 \times 10^{7}$ & 111000 & 16200 & $3.9(1.5)$ \\
$p_{T}>300 \mathrm{GeV}$ & 171000 & 11000 & 8390 & $20(7.7)$ \\
$m($ jet 1$)<80 \mathrm{GeV}$ & 135000 & 6020 & 6370 & $17(6.5)$ \\
\hline tau veto & 119000 & 5840 & 6370 & $18(7.0)$ \\
$b$-jet veto & 115000 & 5290 & 6320 & $19(7.0)$ \\
\hline
\end{tabular}

Table 6. Same as table 3, but now for the mAMSB benchmark scenario, eq. (5.1). We have assumed an integrated luminosity of only $100 \mathrm{fb}^{-1}$ at $\sqrt{s}=14 \mathrm{TeV}$. Jet1 and jet2 denote the jet with the largest $p_{T}$ and second-largest $p_{T}$, respectively.

obtain a good signal to SUSY background ratio, which is needed for a precise estimation of the $\widetilde{\chi}_{1}^{0} \widetilde{q}_{L} q$ coupling $\lambda$. There are some differences in our analysis to the bino-LSP scenario, eq. (4.1). Instead of an integrated luminosity of $300 \mathrm{fb}^{-1}$, we assume only $100 \mathrm{fb}^{-1}$ to show that less statistics is needed to reconstruct $\lambda$. This is due to the much larger monojet cross section for wino-LSP scenarios; cf. section 3.1. For the same reason the discovery potential is better, i.e. we can investigate a heavier spectrum; see table 5 and table 2 .

The cut flow for the mAMSB benchmark scenario, eq. (5.1), is given in table 6 . The first two cuts are identical to those in table 3 . However, the jet veto is slightly different (third row in table 6). Instead of vetoing additional jets with $p_{T} \mathrm{~s}$ larger than $30 \mathrm{GeV}$, we now relax this cut to $50 \mathrm{GeV}$.

In contrast to the mSUGRA scenario, the SUSY background for our mAMSB benchmark point is dominated by Drell-Yan wino pair production; cf. section 3.2. At the parton level, the event topology is similar to that of the signal and to that of the dominant SM background: two invisible particles recoiling against a single quark or gluon. After initial 


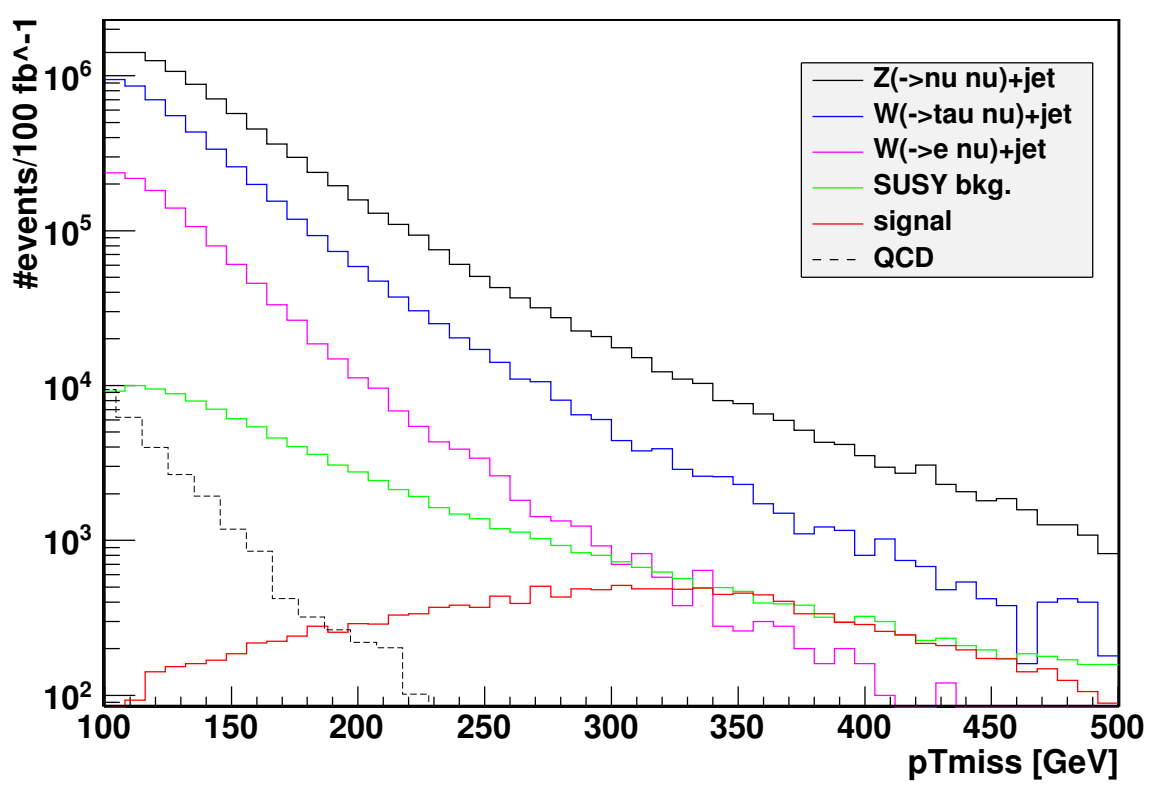

Figure 5. Missing transverse momentum distribution for the $Z(\rightarrow \nu \bar{\nu})+$ jet (black histogram), $W(\rightarrow \tau \nu)+$ jet (blue histogram), $W(\rightarrow e \nu)+$ jet (magenta histogram), the SUSY background (green histogram), the QCD background (black dashed histogram) and the signal process (red histogram). The first three cuts of table 6 have been applied. The number of events correspond to an integrated luminosity of $100 \mathrm{fb}^{-1}$ at $\sqrt{s}=14 \mathrm{TeV}$. We assume the mAMSB scenario of eq. (5.1).

and final state radiation, additional jets can arise and the event might fail the jet veto. The veto then reduces the relative number of signal and $(Z+$ jet and wino-pair plus jet) background events equally due to the similar event topologies. Therefore, the jet veto reduces the significance $S / \sqrt{B}$ whereas the signal to SUSY background ratio stays roughly constant. A weaker jet veto thus increases the visibility of the signal. Note that for the mSUGRA scenario, eq. (4.1), the topology of most of the SUSY background is different. For example, in the mSUGRA scenario, the dominant background is squark pair production, where both squarks decay into a quark and a $\widetilde{\chi}_{1}^{0}$.

After the jet veto, we still observe in table 6 an overwhelming SUSY background, i.e. the signal to SUSY background ratio is roughly seven whereas for our mSUGRA benchmark point this ratio was less than two; see table 3. However a great improvement is possible with a cut on the $\not_{T}$. The relevant distributions (after the first three cuts in table 6 were applied) are shown in figure 5. Here, the SUSY background distributions as well as the SM background distributions fall off exponentially. In contrast, the $\not_{T}$ distribution of the SUSY background in figure 3 does not show the same exponential fall-off; a clear sign that the SUSY background is different in the mSUGRA and mAMSB cases. Applying again a lower $\not p_{T}$ cut around the Jacobian peak of the signal (the red histogram in figure 5) increases the signal to SUSY background ratio to 0.8 and the optimistic (conservative) significance to 20 (7.7). Note that we have also applied the same cut on the $p_{T}$ of the hardest jet. We also see that for this point, the required level of cancellation of the SM 


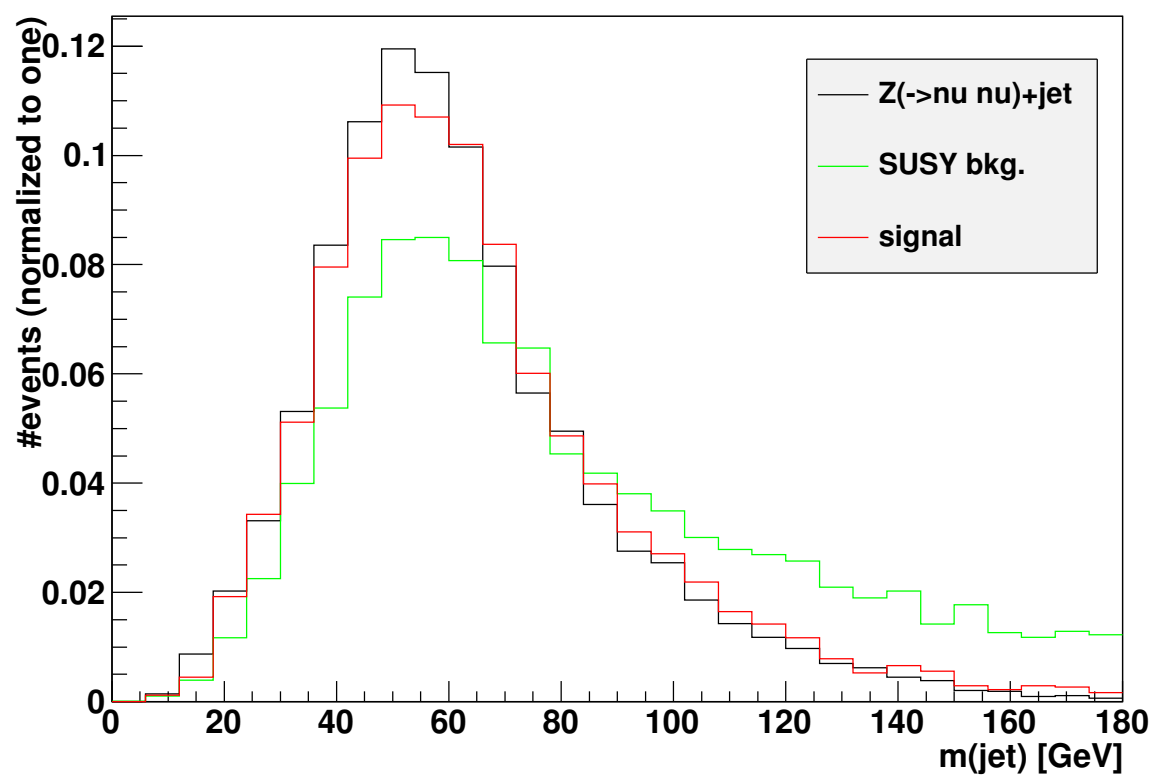

Figure 6. Invariant mass distribution of the hardest jet for the $Z+$ jet background (black histogram), the SUSY background (green histogram) and the signal process (red histogram). The first four cuts of table 6 have been applied. The distributions are normalized to one. For the signal, we assume the mAMSB scenario of eq. (5.1). The QCD background has a distribution almost indistinguishable from that of $Z(\rightarrow \nu \bar{\nu})+$ jet.

model backgrounds is at the $\sim 10 \%$ (or larger) level. Deriving a systematic error smaller than this on the backgrounds measurement ought to be easily achievable with the high number of data expected.

In figure 6 , we present the invariant mass distribution of the hardest jet after the first four cuts of table 6 were applied. Because the SUSY background is dominated by DrellYan like wino pair production, the $m$ (jet1) distribution is less flat than those in figure 4 . However, an improvement with an upper cut on $m$ (jet1) is still possible. Demanding $m($ jet 1$)<80 \mathrm{GeV}$ increases the signal to SUSY background ratio to 1.1. At the same time, the signal significance is not significantly changed. It is 17 (6.5) for our optimistic (conservative) estimate. We finally show in the last two columns of table 6 the effect of a tau and $b$-jet veto. Again, we observe no great improvement by such a veto and thus we shall not apply it in the following.

\subsection{Coupling reconstruction}

We now show to which precision the $\widetilde{\chi}_{1}^{0} \widetilde{q}_{L} q$ coupling $\lambda$ of the mAMSB benchmark scenario of section 5.1 can be reconstructed with an integrated luminosity of $100 \mathrm{fb}^{-1}$ at $\sqrt{s}=14 \mathrm{TeV}$. We employ the cuts given in section 5.2 without the tau and $b$-jet veto. We will closely follow the procedure employed in section 4.3. We reconstruct $\lambda$ under the assumption of the SUSY backgrounds of our mAMSB point. We exhibit in table 7 the statistical and the most important systematic errors for a measurement of the cross section and thus the $\widetilde{\chi}_{1}^{0} \widetilde{q}_{L} q$ coupling. 


\begin{tabular}{|l|cc|}
\hline error & $\Delta \sigma_{\text {mono }} / \sigma_{\text {mono }}$ & $\Delta \lambda / \lambda$ \\
\hline luminosity & $3 \%$ & $1.5 \%$ \\
PDF uncertainty & $17 \%$ & $8.3 \%$ \\
NLO corrections & $18 \%$ & $9 \%$ \\
sparticle mass $\Delta \tilde{m}=10 \mathrm{GeV}$ & $7.3 \%$ & $3.7 \%$ \\
statistics (optimistic) & $5.8 \%$ & $2.9 \%$ \\
statistics (conservative) & $15 \%$ & $7.7 \%$ \\
\hline total (optimistic) & $26 \%$ & $13 \%$ \\
total (conservative) & $30 \%$ & $15 \%$ \\
\hline
\end{tabular}

Table 7. Relative errors for the signal monojet cross section (second column) and the $\widetilde{\chi}_{1}^{0} \widetilde{q}_{L} q$ coupling (third column) from different sources (first column). The numbers are for the mAMSB benchmark scenario, eq. (5.1).

Note that errors from the SUSY background are not included. For our mAMSB benchmark scenario (and for many wino LSP scenarios in general), the SUSY background is dominated by wino pair production plus jet. The differential distribution as a function of the missing transverse momentum is known [40]. One can also measure the SUSY background below the Jacobian peak of the monojet distribution. For our mAMSB benchmark scenario, we found that the SUSY background in an $\not{ }_{T}$ interval of $150 \mathrm{GeV}$ to $300 \mathrm{GeV}$ is more than six times larger than the monojet signal as can be seen in figure 5 . At the same time, for the total number of SUSY events we found a (conservative) significance over SM background of roughly ten. Therefore, it should be possible to estimate the SUSY background from data which reduces significantly the systematic uncertainties of the SUSY background. Assumptions about the underlying model can further reduce this error. We thus expect that errors from SUSY background will not significantly alter the results in table 7. However, a precise reconstruction of the SUSY background lies beyond the scope of this publication.

As in section 4.3 the largest errors come from the PDF uncertainty, from the NLO corrections and from the SM statistics (conservative estimate). There is a good chance that the PDF uncertainty will decrease after LHC has analyzed some first data. Also the calculations of some of the unknown higher order corrections might be performed in the future. Finally, increasing the integrated luminosity would decrease the statistical error. In contrast to the bino LSP scenario, where we already assumed an integrated luminosity of $300 \mathrm{fb}^{-1}$, the coupling reconstruction is possible earlier for our wino LSP scenario, because we only assumed $100 \mathrm{fb}^{-1}$ of data. For example, assuming an integrated luminosity of $300 \mathrm{fb}^{-1}$ and an error from the PDFs and from higher order corrections of $10 \%$, the relative error of $\lambda$ would decrease from $13 \%$ (15\%) in table 7 to $8.3 \%$ (9.2\%) for the optimistic (conservative) statistical estimate. To conclude, a measurement of $\lambda$ with a precision of roughly $10 \%$ at the end of LHC running seems to be possible for our mAMSB benchmark scenario. 


\subsection{Reconstruction of couplings under the assumption of a wino LSP}

We have seen that the reconstruction of the coupling $\lambda$ is much more promising in SUSY scenarios with a wino like LSP than with a bino like LSP. Therefore, we will henceforth concentrate in this section on wino LSP scenarios. We will investigate the precision to which $\lambda$ can be measured as a function of the (left-handed) squark mass and the $\widetilde{\chi}_{1}^{0}$ mass.

For that purpose, we perform a two-dimensional parameter scan. We have varied the pole masses of the left-handed first generation squarks (winos) between $400 \mathrm{GeV}$ and $1210 \mathrm{GeV}(100 \mathrm{GeV}$ and $400 \mathrm{GeV})$. All other SUSY particles are decoupled. This part of parameter space has the chargino-squark-quark couplings equal to $\lambda=g$ to better than per mille precision. We employ a grid of ten times ten points equally spaced in the squark-wino mass plane. We assumed an integrated luminosity of $100 \mathrm{fb}^{-1}$ at $\sqrt{s}=14 \mathrm{TeV}$. For the relative error from the luminosity we use again $3 \%$, whereas the errors from PDFs, NLO corrections, and the SUSY mass uncertainties were estimated in the following way. We calculated for a sub-grid with four times four parameter points the respective errors as it was done in section 5.3 and extrapolated the errors for the other parameter points.

The PDF uncertainties are described well by a linear function of the squark and $\widetilde{\chi}_{1}^{0}$ mass sum in which the error decreases with increasing mass. This behaviour can be understood by noting that the error of the gluon PDF decreases in the relevant region for increasing Bjorken- $x$ [92]. For the NLO uncertainties we use a logarithmic fit as a function of the sum of the squark and $\widetilde{\chi}_{1}^{0}$ mass. Prospino employs the squark and $\widetilde{\chi}_{1}^{0}$ mass sum divided by two as its default factorization and renormalization scale. For the scale variation both scales are then varied between $1 / 2$ and 2 times the default value, cf. section 4.3. A logarithmic behaviour is expected, because the scale dependence enters via loops.

The estimate of the error from SUSY mass uncertainties is more involved. In general, the precision to which the masses can be reconstructed depends on the nature of the SUSY particle, the mass spectrum and the model assumptions; see for example refs. [4, 101]. We here employ a simple approach. We assume that a squark mass of $m_{\tilde{q}_{L}}=720 \mathrm{GeV}$ can be reconstructed with an absolute error of $\delta m_{\tilde{q}_{L}}=10 \mathrm{GeV}$. For a $\tilde{\chi}_{1}^{0}$ with a mass of $100 \mathrm{GeV}$ we assume the same error. This corresponds to our assumption made in section 5.3. We then estimated the error for other squark masses $m_{\tilde{q}_{L}}^{\prime}$ via

$$
\delta m_{\tilde{q}_{L}}^{\prime}=\delta m_{\tilde{q}_{L}} \times \frac{m_{\tilde{q}_{L}}^{\prime}}{m_{\tilde{q}_{L}}} \times \sqrt{\frac{\sigma\left(P P \rightarrow \widetilde{q}_{L} \widetilde{q}_{L}\right)}{\sigma\left(P P \rightarrow \widetilde{q}_{L}^{\prime} \widetilde{q}_{L}^{\prime}\right)}},
$$

i.e. the relative error scales with the square root of the inverse squark pair production cross section, $\sigma\left(P P \rightarrow \widetilde{q}_{L} \widetilde{q}_{L}\right)$, as expected if the errors are dominated by statistical uncertainties and if the masses are reconstructed from cascade decays of squarks.

We also employ the same absolute error for the $\widetilde{\chi}_{1}^{0}$ mass, because on the one hand, the squark and $\widetilde{\chi}_{1}^{0}$ mass error are always strongly correlated to each other if the squark decays into the $\widetilde{\chi}_{1}^{0}$ plus a jet [101]. On the other hand, analysis like for the benchmark point SPS1a [102] show that the lightest neutralino mass can be slightly better reconstructed than the squark masses $[4,101]$. We thus follow here a conservative approach. From the mass errors we obtain errors for the total signal cross section as it was done in section 5.3. 

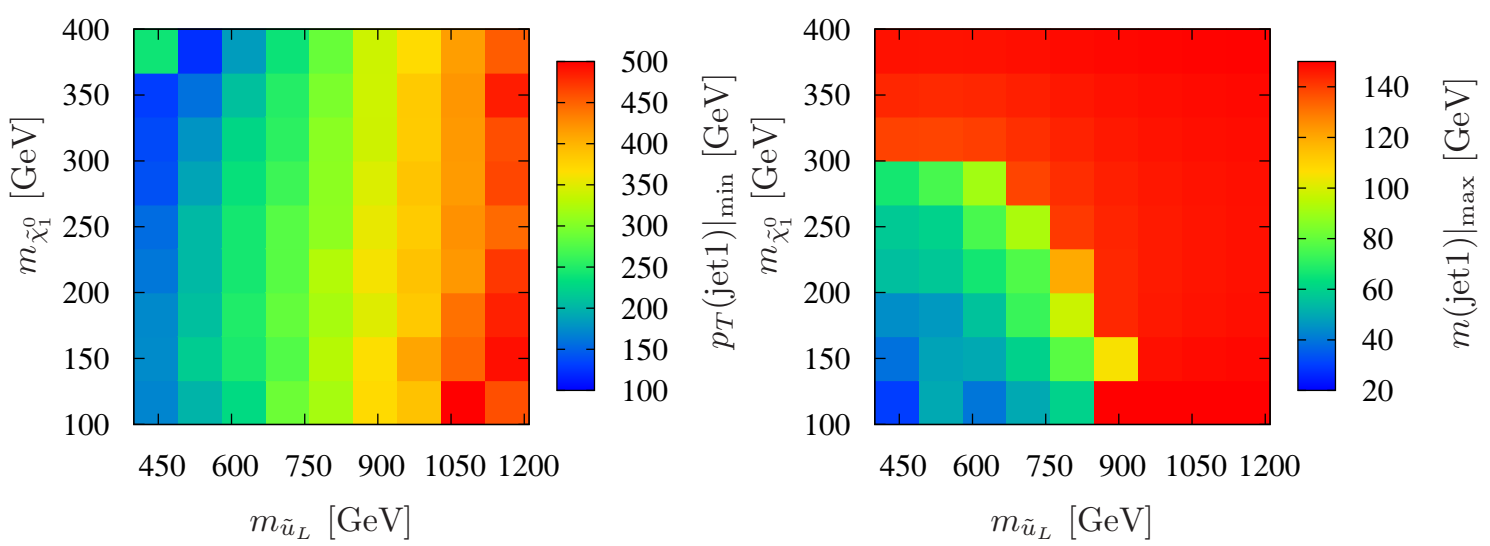

Figure 7. Lower cut for the jet- $p_{T}$ (left-hand side) and upper cut for the jet invariant mass (righthand side) as a function of the squark and $\widetilde{\chi}_{1}^{0}$ mass. The red region in the right figure corresponds to no cut on $m($ jet 1$)$.

We found that a quadratic fit as a function of the squark mass describes very well the calculated errors as might be expected from eq. (5.2).

In order to find the right cuts for each SUSY scenario, we have employed a similar strategy to the one in section 5.3. We applied the first three cuts of table 6. Starting from the trigger cut of $100 \mathrm{GeV}$, we searched numerically in steps of $10 \mathrm{GeV}$ for a (lower) $p_{T}$ cut on the hardest jet that maximizes the significance $S / \sqrt{B}$, where $S(B)$ corresponds to the number of signal (SM background events). Finally, we varied the (upper) cut on the invariant mass of the hardest jet in steps of $10 \mathrm{GeV}$ in order to maximize the signal to SUSY background ratio. However, we only employ a cut on the invariant mass as long as the (conservative) significance is larger than five.

The resulting cuts employed are shown in figure 7 for the jet- $p_{T}$ (which is equal to the cut on $\not p_{T}$ ) and for the jet invariant mass. They are given as a function of the left-handed up-squark mass, $m_{\tilde{u}_{L}}$, and the (wino-like) $\widetilde{\chi}_{1}^{0}$ LSP mass, $m_{\tilde{\chi}_{1}^{0}}$. Note that the left-handed down squark and left-handed up squark are quasi-degenerate, as are the $\widetilde{\chi}_{1}^{+}$and $\widetilde{\chi}_{1}^{0}$. We can see that the lower cut on the jet- $p_{T}$ increases with increasing squark mass and decreasing $\widetilde{\chi}_{1}^{0}$ mass as expected from the position of the Jacobian peak given in eq. (1.1). Recall that we usually obtain the best significance if we apply the jet- $p_{T}$ cut close to the Jacobian peak; cf. sections 4.2 and 5.2 .

However, an exception is the quite large jet- $p_{T}$ cut in the upper left corner of figure 7 . Due to the small squark- $\widetilde{\chi}_{1}^{0}$ mass difference, the Jacobian peak lies below $\lesssim 100 \mathrm{GeV}$, i.e below the trigger cut. For larger $p_{T}$ values (compared to the peak), the jet- $p_{T}$ (and $\not_{T}$ ) distribution of the signal falls off less steep than the SM backgrounds. Therefore, for a small squark- $\widetilde{\chi}_{1}^{0}$ mass difference the best cut will lie above the Jacobian peak.

Figure 7 (right side) shows the upper cut on the invariant mass of the monojet. The red region corresponds to parameter points, where the conservative significance, $S / \sqrt{7 B}$, is smaller than five. In this case, we do not apply a cut on the invariant jet mass in order not to suppress the signal cross section. We observe that harder cuts on the invariant mass are usually applied for smaller squark and $\widetilde{\chi}_{1}^{0}$ masses, because the number of signal events 


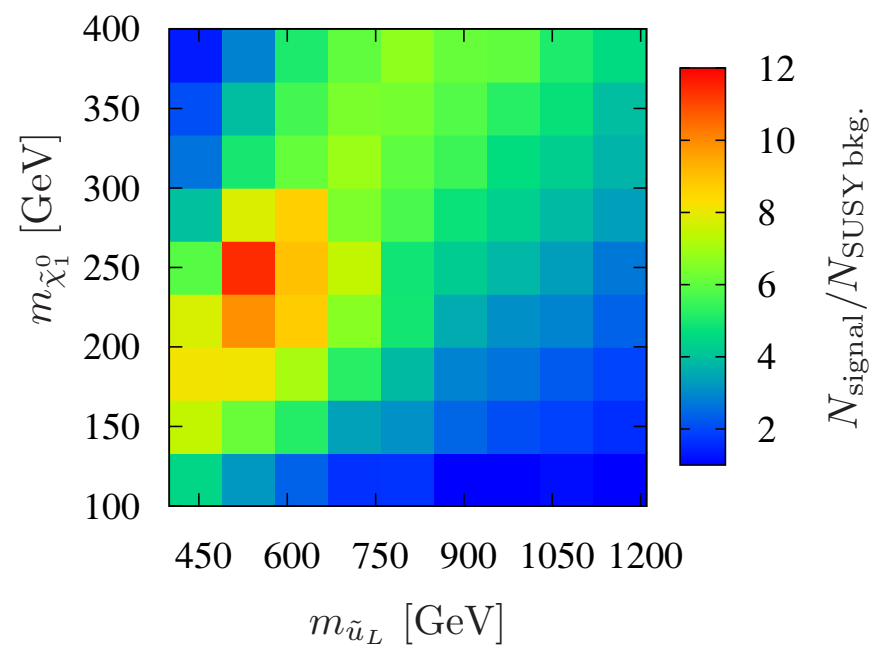

Figure 8. Ratio of the number of signal events to the number of SUSY background events as a function of the squark and $\widetilde{\chi}_{1}^{0}$ mass.

is large and a hard cut will not suppress the signal below the discovery reach. Recall from figure 4 and figure 6 that harder cuts on the invariant mass lead often to a better signal to SUSY background ratio.

The importance of the invariant mass cut can be seen in figure 8 , where the ratio of signal to SUSY background events (after cuts) as a function of the squark and $\widetilde{\chi}_{1}^{0}$ mass is shown. We observe that the ratio varies between 1.2 and 12. This good signal to SUSY background ratio justifies our approximation to neglect the (unknown) error from SUSY backgrounds as long as the SUSY background can be determined to a precision of a few 10\%; see also the discussion in section 4.3 and section 5.3.

We also see some interesting structure in figure 8: for fixed squark mass, the signal to SUSY background ratio first increases when we increase the $\widetilde{\chi}_{1}^{0}$ mass and then decreases again. For small $\widetilde{\chi}_{1}^{0}$ mass, the SUSY background is dominated by Drell-Yan wino pair production. However, when the $\widetilde{\chi}_{1}^{0}$ mass is increased the respective cross section will decrease and squark pair production will take over to be the dominant background. At the same time, as long as the $\widetilde{\chi}_{1}^{0}$ mass increases moderately (relative to the squark mass) the signal cross section decreases less fast than the SUSY background leading to a better signal to SUSY background ratio. However, when we come to the region where $m_{\tilde{\chi}_{1}^{0}} \approx m_{\tilde{q}_{L}}$ we obtain a reduced signal to SUSY background ratio. The signal to SUSY background ratio can be further increased with a cut on the jet invariant mass. As can be seen in figure 8, this can result in a signal to SUSY background ratio larger than ten!

The fractional precision that can be achieved can be seen in figure 9, where we show the relative error of the coupling $\lambda$ as a function of the left-handed up-squark mass and the $\widetilde{\chi}_{1}^{0}$ LSP mass. Fractional precisions between 0.12 and 0.24 are possible throughout parameter space for optimistic assumptions, or $0.12-0.44$ for conservative ones. The most precise measurements are for lighter sparticles, where signal event numbers are higher.

The significance of the SUSY monojet signal is also given in figure 9 by the solid 


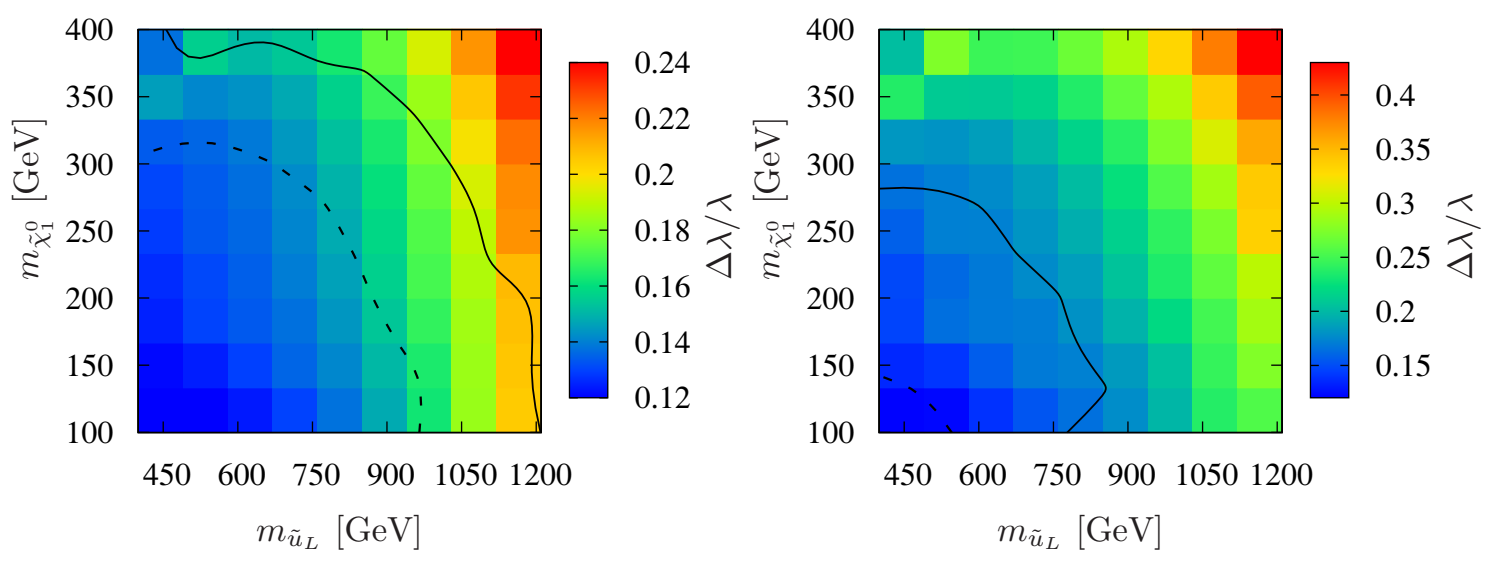

Figure 9. Fractional precision to which the $\widetilde{\chi}_{1}^{0} \widetilde{q}_{L} q$ coupling $\lambda$ can be reconstructed as function of the squark and $\widetilde{\chi}_{1}^{0}$ mass. The left (right) figure employs our optimistic (conservative) estimate for the SM background uncertainties. The solid and dashed black lines correspond to $S / \sqrt{B}(S / \sqrt{7 B})$ of $5 \sigma$ and $10 \sigma$, respectively.

(dashed) black line corresponding to the $5 \sigma(10 \sigma)$ region. We see a strong dependence on whether we assume optimistic or conservative error estimates. Whether or not the signal can be seen over statistical fluctuations of the background to the $5 \sigma$ level, the coupling $\lambda$ may be bounded.

To conclude, the LHC will be able to test the $\widetilde{\chi}_{1}^{0} \widetilde{q}_{L} q$ coupling relation to a precision of $\mathcal{O}(10 \%)$ in large regions of the wino LSP parameter space as long as the squark masses are $\lesssim 1 \mathrm{TeV}$.

\section{Summary and conclusion}

If signatures of new physics beyond the SM are discovered that are consistent with SUSY, a program of measurements of the interactions of new states would be useful to hypothesis test the SUSY interpretation and cross-correlate with other measurements. As a first step, we have investigated the feasibility of SUSY monojet production at the LHC via $q g \rightarrow \widetilde{q} \widetilde{\chi}_{1}^{0} \rightarrow q \widetilde{\chi}_{1}^{0} \widetilde{\chi}_{1}^{0}$ for measuring the $\widetilde{\chi}_{1}^{0} \widetilde{q} q$ coupling $\lambda$, under the assumption that the $\widetilde{\chi}_{1}^{0}$ is the LSP.

For a bino LSP, supersymmetry predicts that $\lambda$ is proportional to $g^{\prime}$, the $\mathrm{U}(1)_{Y}$ gauge coupling. For a wino LSP however, supersymmetry predicts $\lambda=g$. When added to other empirical information on the field content of the neutralino, the value of the coupling constitutes a test of supersymmetry. In general, the MSSM $\widetilde{\chi}_{1}^{0}$ contains admixtures of the wino, the bino and the higgsino, resulting in $\lambda$ being a mixture of $g, g^{\prime}$ and 0 , respectively. Without additional information on the field content coming from other measurements, a measurement of the coupling of the LSP to a quark and a squark (in the framework of the MSSM) yields valuable information regarding the neutralino field content. As such, it could be used in the calculation of its thermal relic density in the universe, or for dark matter direct detection rates, for example. Our signal would only be measured significantly above backgrounds after data have been amassed for many years at the LHC. Therefore it 
is reasonable to expect that information on the mass spectrum would already be known, e.g. squark and neutralino mass scales, thereby constraining the SUSY backgrounds.

At this stage, it would be useful to extract, as far as possible, SUSY backgrounds from other data samples. However, without data to guide us, the number of possibilities is very large. The most difficult part of estimating SUSY backgrounds would likely be the extraction of the relevant branching ratio for the $\widetilde{q} \rightarrow q \widetilde{\chi}_{1}^{0}$ decay. Nevertheless, placing an upper bound of 1 on the branching ratio for an observed SUSY monojet signal would yield a lower bound on $\lambda$. If the LSP is a generic admixture of bino-wino-higgsino eigenstates, one must also take into account the fact that the lightest chargino may couple differently to the lightest neutralino. However, one would then generically expect the $\widetilde{\chi}_{1}^{ \pm}$to not be quasi-mass degenerate with the $\widetilde{\chi}_{1}^{0}$, and so its decays ought to be visible and therefore reducible by our cuts. On the other hand, it may be that a simple model of supersymmetry breaking with only a few parameters is selected by fitting all available data. In this case, the branching ratio and therefore the total rate of our monojet signal would be predicted and would constitute an additional hypothesis test on that model.

Measuring $\lambda$ is not easy at the LHC, but we have shown that a fractional accuracy approaching $10 \%$ is feasible with a large data set, providing a non-trivial and useful empirical constraint on the MSSM. As such, it will be an important element of the proposed program of precision measurements of new particle interactions to support the SUSY hypothesis.

\section{Acknowledgments}

B.C.A. would like to thank Mihoko Nojiri for early discussions and her kind help with event generation, other members of the Cambridge SUSY working group, Alan Barr, Jon Butterworth and Kyle Cranmer for comments and suggestions. H.E.H. appreciates useful conversations with Tilman Plehn, Herbi Dreiner and JoAnne Hewett. S.G. thanks Michael Kraemer, Jong Soo Kim, Sebastian Fleischmann and Peter Wienemann for helpful discussions.

B.C.A. is supported in part by a grant from STFC and an associateship from the IPPP in Durham. The work of S.G. is supported in part by the U.S. Department of Energy, under grant number DE-FG02-04ER41268 and in part by a Feodor Lynen Research Fellowship sponsored by the Alexander von Humboldt Foundation. H.E.H. is supported in part by the U.S. Department of Energy, under grant number DE-FG02-04ER41268 and in part by a Humboldt Research Award sponsored by the Alexander von Humboldt Foundation.

\section{A The Jacobian peak in the transverse momentum distribution}

The Jacobian peak is a well-known feature of the transverse momentum distribution of the electron in the process $A+B \rightarrow W^{ \pm}+X \rightarrow e^{ \pm}+\nu+X$, where $A$ and $B$ are the initial state hadrons. The resulting peak at $p_{T} \simeq \frac{1}{2} m_{W}$ is a consequence of the Jacobian that arises from changing kinematic variables from $\cos \theta$ (where $\theta$ is the center-of-mass scattering angle) to $p_{T} \cdot{ }^{10}$ In this paper, we have focused on monojets that arise from $\widetilde{q}_{1}^{0}$ production, where

\footnotetext{
${ }^{10}$ For a pedagogical treatment, see ref. [103].
} 
$\widetilde{q} \rightarrow q \widetilde{\chi}_{1}^{0}$, and the quark is observed as a hadronic jet. The $p_{T}$ distribution of the quark jet also exhibits a Jacobian peak. In this appendix, we derive an approximate expression for the location of the peak in the transverse momentum distribution of the jet.

Consider the $2 \rightarrow 3$ scattering process, which schematically is of the form:

$$
a+b \rightarrow c+3, \text { followed by } c \rightarrow 1+2,
$$

where the decaying particle $c$ is spinless. Since the particles $a, b$ and 1 represent light quarks or gluons, we shall set their masses to zero, $m_{a}=m_{b}=m_{1}=0$. We denote the mass of particle $c$ (identified as the $\widetilde{q}$ ) to be $m_{c} \equiv M$, and the masses of particles 2 and 3 (which are identified with $\widetilde{\chi}_{1}^{0}$ ) to be $m_{2}=m_{3} \equiv m$.

If the particle $c$ is on-shell, then the corresponding matrix element for the $2 \rightarrow 2$ process, $a+b \rightarrow c+3$ is of the form

$$
\mathcal{M}(a+b \rightarrow c+3)=C_{1}(s, t),
$$

where $C_{1}(s, t)$ is a dimensionless function of $s \equiv\left(p_{a}+p_{b}\right)^{2}, t \equiv\left(p_{a}-p_{1}\right)^{2}$ and the particle masses. For the decay of particle $c$ (which is either $\widetilde{q}_{L}$ or $\widetilde{q}_{R}$ ), the squared matrix element, summed over final spins, is given by

$$
|\mathcal{M}(c \rightarrow 1+2)|^{2}=C_{2}\left(M^{2}-m^{2}\right),
$$

where $C_{2}$ is a dimensionless (real positive) constant that will eventually cancel out in our computation. Using, eq. (A.3) it follows that the total width of particle $c$ times the branching ratio is given by

$$
B \Gamma=\frac{C_{2} M}{16 \pi}\left(1-\frac{m^{2}}{M^{2}}\right)^{2}
$$

where $B \equiv B(c \rightarrow 1+2)$.

To set up our computation, we work in the center-of-mass system. Then, the fourvectors of the initial states and the observed final state (particle 1) are:

$$
\begin{aligned}
p_{a} & =\frac{1}{2} \sqrt{s}(1 ; 0,0,1), \\
p_{b} & =\frac{1}{2} \sqrt{s}(1 ; 0,0,-1), \\
p_{1} & =E_{1}(1 ; \sin \theta, 0, \cos \theta),
\end{aligned}
$$

where $\theta$ is the scattering angle in the center-of-mass frame. Following ref. [104], we define three invariants,

$$
\begin{aligned}
& t_{1} \equiv t=\left(p_{a}-p_{1}\right)^{2}=-\sqrt{s} E_{1}(1-\cos \theta) \\
& s_{1} \equiv p_{c}^{2}=\left(p_{1}+p_{2}\right)^{2} \\
& s_{2} \equiv\left(p_{a}+p_{b}-p_{1}\right)^{2}=s-2 \sqrt{s} E_{1} .
\end{aligned}
$$

We denote the three-body phase space integral by

$$
R_{3}(s)=\int \prod_{i=1}^{3} \frac{d^{3} p_{i}}{2 E_{i}} \delta^{(3)}\left(\boldsymbol{p}_{\boldsymbol{a}}+\boldsymbol{p}_{b}-\boldsymbol{p}_{1}-\boldsymbol{p}_{2}-\boldsymbol{p}_{3}\right) \delta\left(\sqrt{s}-E_{1}-E_{2}-E_{3}\right) .
$$


The key formula that we need is given by eq. V-7.8 of ref. [104],

$$
\begin{aligned}
\frac{d R_{3}}{d s_{2} d t_{1} d s_{1}}=\frac{\pi^{2}}{4 \lambda^{1 / 2}\left(s, m_{a}^{2}, m_{b}^{2}\right) \lambda^{1 / 2}\left(s, s_{2}, m_{1}^{2}\right)} & \Theta\left\{-G\left(s, t_{1}, s_{2}, m_{a}^{2}, m_{b}^{2}, m_{1}^{2}\right)\right\} \\
& \times \Theta\left\{-G\left(s_{1}, s_{2}, s, m_{2}^{2}, m_{1}^{2}, m_{3}^{2}\right)\right\},
\end{aligned}
$$

where

$$
\lambda(a, b, c) \equiv a^{2}+b^{2}+c^{2}-2 a b-2 a c-2 b c
$$

is the well-known triangle function of relativistic kinematics, and $G$ is the basic four-particle kinematic function, first introduced in ref. [105, 106]

$$
G(x, y, z, u, v, w) \equiv-\frac{1}{2} \operatorname{det}\left(\begin{array}{ccc}
2 u & x+u-v & u+w-y \\
x+u-v & 2 x & x-z+w \\
u+w-y & x-z+w & 2 w
\end{array}\right) .
$$

Expanding out the determinant yields the unwieldy expression, ${ }^{11}$

$$
\begin{aligned}
G(x, y, z, u, v, w)= & x y(x+y)+z u(z+u)+v w(v+w)+x(z w+u v)+y(z v+u w) \\
& -x y(z+u+v+w)-z u(x+y+v+w)-v w(x+y+z+u) .
\end{aligned}
$$

In obtaining eq. (A.12), we have integrated over an unobserved azimuthal angle. ${ }^{12}$ The theta functions above determine the kinematical ranges of the parameters $s_{1}, s_{2}$ and $t_{1}$. Taking $m_{a}=m_{b}=m_{1}=0$ and $m_{2}=m_{3}=m$, it follows that:

$$
\frac{d R_{3}}{d s_{2} d t_{1} d s_{1}}=\frac{\pi^{2}}{4 s\left(s-s_{2}\right)} \Theta\left\{-G\left(s, t_{1}, s_{2}, 0,0,0\right)\right\} \Theta\left\{-G\left(s_{1}, s_{2}, s, m^{2}, 0, m^{2}\right)\right\} .
$$

The differential cross-section is given by:

$$
d \sigma=\frac{1}{64 \pi^{5} s} d R_{3}(s)|\mathcal{M}(a+b \rightarrow 1+2+3)|^{2},
$$

where the squared matrix element is suitably averaged over initial spins and summed over final spins. The dominant contribution to $a+b \rightarrow 1+2+3$ takes place via $a+b \rightarrow c+3$, where $c$ is produced approximately on-shell and subsequently decays via $c \rightarrow 1+2$. In particular, since $c$ is a spin-zero particle,

$$
|\mathcal{M}(a+b \rightarrow 1+2+3)|^{2} \simeq \frac{|\mathcal{M}(a+b \rightarrow c+3)|^{2}|\mathcal{M}(c \rightarrow 1+2)|^{2}}{\left(s_{1}-M^{2}\right)^{2}+M^{2} \Gamma^{2}} .
$$

We now use eqs. (A.2) and (A.3) and employ the narrow width approximation,

$$
\frac{1}{\left(s_{1}-M^{2}\right)^{2}+M^{2} \Gamma^{2}} \longrightarrow \frac{\pi}{M \Gamma} \delta\left(s_{1}-M^{2}\right) .
$$

\footnotetext{
${ }^{11}$ Eq. (A.15), which is first defined in ref. [105, 106], is also given in eq. IV-5.23 of ref. [104]. We have noted a typographical error in the latter; in the second line of eq. IV-5.23, the first term $y z w$ should read $y z v$.

${ }^{12}$ This is the so-called helicity angle, which is defined as the azimuthal angle between the production plane spanned by $\overrightarrow{\boldsymbol{p}}_{b}$ and $\overrightarrow{\boldsymbol{p}}_{1}$ and the plane spanned by $\overrightarrow{\boldsymbol{p}}_{1}$ and $\overrightarrow{\boldsymbol{p}}_{3}$ with $\overrightarrow{\boldsymbol{p}}_{1}$ as the axis, in a reference frame where $\overrightarrow{\boldsymbol{p}}_{2}+\overrightarrow{\boldsymbol{p}}_{3}=\overrightarrow{\boldsymbol{p}}_{a}+\overrightarrow{\boldsymbol{p}}_{b}-\overrightarrow{\boldsymbol{p}}_{1}=0$. By convention, the production plane is taken to be the $x-z$ plane. See ref. [104] for further details.
} 
Hence, it follows that:

$$
\begin{aligned}
\frac{d \sigma}{d s_{2} d t_{1} d s_{1}}= & \frac{B\left|C_{1}\left(s, t_{1}\right)\right|^{2}}{16 \pi \xi s^{2}\left(s-s_{2}\right)} \delta\left(s_{1}-M^{2}\right) \\
& \times \Theta\left\{-G\left(s, t_{1}, s_{2}, 0,0,0\right)\right\} \Theta\left\{-G\left(s_{1}, s_{2}, s, m^{2}, 0, m^{2}\right)\right\},
\end{aligned}
$$

where

$$
\xi \equiv 1-\frac{m^{2}}{M^{2}}
$$

Assuming that $G\left(s_{1}, s_{2}, s, m^{2}, 0, m^{2}\right)<0$, we can immediately use the $\delta$-function to integrate over $s_{1}$. Using eqs. IV-5.28 and IV-5.29 of ref. [104],

$$
G\left(s_{1}, s_{2}, s, m^{2}, 0, m^{2}\right)=s_{2}\left(s_{1}-s_{1}^{+}\right)\left(s_{1}-s_{1}^{-}\right),
$$

where $s_{2}$ is strictly non-negative and

$$
s_{1}^{ \pm}=m^{2}+\frac{1}{2}\left(s-s_{2}\right)\left[1 \pm \sqrt{1-\frac{4 m^{2}}{s_{2}}}\right] .
$$

That is, we require that:

$$
s_{1}^{-} \leq M^{2} \leq s_{1}^{+},
$$

otherwise, $s_{1}=M^{2}$ can never be satisfied when $G\left(s_{1}, s_{2}, s, m^{2}, 0, m^{2}\right)<0$. Inserting eq. (A.23) into eq. (A.24), one obtains upper and lower limits for $s_{2}$. One can then use eq. (A.10) to obtain upper and lower limits for $E_{1}$. These limits correspond to the roots of the quadratic equation,

$$
4 \sqrt{s} M^{2} E_{1}^{2}-2\left(M^{2}-m^{2}\right)\left(s+M^{2}-m^{2}\right) E_{1}+\sqrt{s}\left(M^{2}-m^{2}\right)^{2}=0 .
$$

The roots can be expressed as: ${ }^{13}$

$$
E_{1}^{ \pm} \equiv \frac{\xi}{4 \sqrt{s}}\left[s+M^{2}-m^{2} \pm \lambda^{1 / 2}\left(s, M^{2}, m^{2}\right)\right]
$$

where $\xi$ is defined in eq. (A.21). Likewise, employing eq. (A.10), we define

$$
s_{2}^{ \pm}=s-2 \sqrt{s} E_{1}^{\mp} .
$$

We now integrate eq. (A.20) over $s_{1}$, under the assumption that $s_{2}^{-} \leq s_{2} \leq s_{2}^{+}$. Then,

$$
\frac{d \sigma}{d s_{2} d t_{1}}=\frac{B\left|C_{1}\left(s, t_{1}\right)\right|^{2}}{16 \pi \xi s^{2}\left(s-s_{2}\right)} \Theta\left\{-G\left(s, t_{1}, s_{2}, 0,0,0\right)\right\} .
$$

The range of $t_{1}$ is determined from the inequality:

$$
G\left(s, t_{1}, s_{2}, 0,0,0\right) \equiv s t_{1}\left(s+t_{1}-s_{2}\right) \leq 0,
$$

\footnotetext{
${ }^{13}$ Note that eq. (A.26) is equivalent to $E_{1}^{ \pm}=\frac{1}{2} \xi\left(E_{c} \pm p_{c}\right.$ ), where $E_{c}$ and $p_{c}$ are the center-of-mass energy and momentum of the decaying particle $c$.
} 
where we have used eq. (A.15) to evaluate the $G$-function. That is,

$$
s_{2}-s \leq t_{1} \leq 0,
$$

as $s_{2}$ ranges over $s_{2}^{-} \leq s_{2} \leq s_{2}^{+}$.

We now introduce the transverse momentum, $p_{T}$ of particle 1 , which is defined by $p_{T}=E_{1} \sin \theta$. It then follows that:

$$
\cos \theta= \pm \sqrt{1-\frac{p_{T}^{2}}{E_{1}^{2}}}
$$

where the \pm indicates that $\theta$ and $\pi-\theta$ correspond to the same value of $p_{T}$. It follows that:

$$
t_{1}=-\sqrt{s}\left(E_{1}-\sqrt{E_{1}^{2}-p_{T}^{2}}\right) .
$$

We now perform a change of variables from $\left\{t_{1}, s_{2}\right\}$ to $\left\{p_{T}^{2}, E_{1}\right\}$. Computing the Jacobian of the transformation, it follows that:

$$
d t_{1} d s_{2}=\frac{s d p_{T}^{2} d E_{1}}{\sqrt{E_{1}^{2}-p_{T}^{2}}} .
$$

The limits of the kinematic variables $p_{T}$ and $E_{1}$ are given by:

$$
0 \leq p_{T} \leq E_{1}, \quad E_{1}^{-} \leq E_{1} \leq E_{1}^{+},
$$

where the range of $p_{T}$ follows from $|\cos \theta| \leq 1$ and $E_{1}^{ \pm}$is defined in eq. (A.26). Since we aim to compute $d \sigma / d p_{T}$, it is more useful to interchange the order of integration. Thus, equivalent to eq. (A.34) is:

$$
\begin{array}{ll}
\text { for } \quad 0 \leq p_{T} \leq E_{1}^{-}, & E_{1}^{-} \leq E_{1} \leq E_{1}^{+}, \\
\text {for } \quad E_{1}^{-} \leq p_{T} \leq E_{1}^{+}, & p_{T} \leq E_{1} \leq E_{1}^{+} .
\end{array}
$$

Combining eqs. (A.28) and (A.33), and multiplying the overall result by 2 to take into account the two possible signs of $\cos \theta$ in eq. (A.31),

$$
\frac{d \sigma}{d p_{T}^{2} d E_{1}}=\frac{B\left|C_{1}\left(s, t_{1}\right)\right|^{2}}{16 \pi \xi s^{3 / 2} E_{1} \sqrt{E_{1}^{2}-p_{T}^{2}}},
$$

where $t_{1}$ is expressed in terms of $E_{1}$ and $p_{T}^{2}$ via eq. (A.32). We now integrate over $E_{1}$, employing the limits of integration given in eqs. (A.35) and (A.36). Writing $d p_{T}^{2}=2 p_{T} d p_{T}$, we arrive at our final result, ${ }^{14}$

$$
\frac{d \sigma}{d p_{T}}=\frac{B p_{T}}{8 \pi \xi s^{3 / 2}} \int_{E_{\min }}^{E_{\max }} \frac{d E_{1}}{E_{1} \sqrt{E_{1}^{2}-p_{T}^{2}}}\left|C_{1}\left(s, t_{1}\right)\right|^{2},
$$

\footnotetext{
${ }^{14}$ There is no singularity in the limit of $m \rightarrow M$, since in this limit, $E^{ \pm} \rightarrow 0$.
} 
where $t_{1} \equiv-\sqrt{s}\left[E_{1}-\sqrt{E_{1}^{2}-p_{T}^{2}}\right]$, and the upper and lower limits of integration are given by $E_{\max } \equiv E_{1}^{+}$and

$$
E_{\min }=\left\{\begin{array}{ll}
E_{1}^{-} & \text {for } \quad 0 \leq p_{T} \leq E_{1}^{-} \\
p_{T} & \text { for } \quad E_{1}^{-} \leq p_{T} \leq E_{1}^{+}
\end{array} .\right.
$$

As a warmup, we shall ignore the details of the scattering matrix element for the process $a+b \rightarrow c+3$ by putting $C_{1}=1$. In this case, the integral in eq. (A.38) is elementary, and the end result is:

$$
\frac{d \sigma}{d p_{T}}=\frac{B}{8 \pi \xi s^{3 / 2}}\left[\tan ^{-1}\left(\frac{\sqrt{\left[E_{1}^{+}\right]^{2}-p_{T}^{2}}}{p_{T}}\right)-\Theta\left(E_{1}^{-}-p_{T}\right) \tan ^{-1}\left(\frac{\sqrt{\left[E_{1}^{-}\right]^{2}-p_{T}^{2}}}{p_{T}}\right)\right],
$$

where $0 \leq p_{T} \leq E_{1}^{+}$, and the step function $\Theta$ is defined as usual,

$$
\Theta\left(E_{1}^{-}-p_{T}\right)= \begin{cases}1 & \text { for } \quad 0 \leq p_{T} \leq E_{1}^{-} \\ 0 & \text { for } \quad E_{1}^{-} \leq p_{T} \leq E_{1}^{+}\end{cases}
$$

It is convenient to introduce dimensionless variables,

$$
x \equiv \frac{2 p_{T}}{\sqrt{s}}, \quad y \equiv \frac{M^{2}}{s}, \quad z \equiv 1-\xi=\frac{m^{2}}{M^{2}} .
$$

The kinematics of the scattering process requires that $\sqrt{s} \geq M+m$, which is equivalent to the condition,

$$
\sqrt{y}(1+\sqrt{z}) \leq 1
$$

In this case, the range of the variable $E_{1}$ is given by $E_{1}^{-} \leq E_{1} \leq E_{1}^{+}$, where

$$
E_{1}^{ \pm}=\frac{1}{4}(1-z) \sqrt{s}\left[1+y(1-z) \pm \lambda^{1 / 2}(1, y, y z)\right]
$$

The range of $x$ is now given by:

$$
0 \leq x \leq \frac{1}{2}(1-z)\left[1+y(1-z)+\lambda^{1 / 2}(1, y, y z)\right]<1 .
$$

As an example, take $y=0.5$ and $z=0.1$, which is consistent with the inequality given in eq. (A.43). Eq. (A.45) then implies that $0 \leq x \leq 0.79657$. The transverse momentum distribution, plotted in figure 10 exhibits a striking Jacobian peak, which is located at

$$
\left(p_{T}\right)_{\text {peak }}=E_{1}^{-}
$$

The origin of the Jacobian peak is a consequence of the change in kinematic variables given in eq. (A.33), and is rather insensitive to the form of the matrix element. To illustrate this point, we have numerically evaluated eq. (A.38), where the tree-level form for $C_{1}$ for $g q \rightarrow \widetilde{q}_{R} \widetilde{\chi}_{1}^{0}$ is employed $[11,35,36,62]$

$$
C_{1}(s, t)=N\left[\frac{s+t-M^{2}}{2 s}-\frac{M^{2}\left(m^{2}-t\right)}{\left(M^{2}-t\right)^{2}}+\frac{s m^{2}+\left(m^{2}-t\right)\left(M^{2}-m^{2}\right)}{s\left(M^{2}-t\right)}\right],
$$




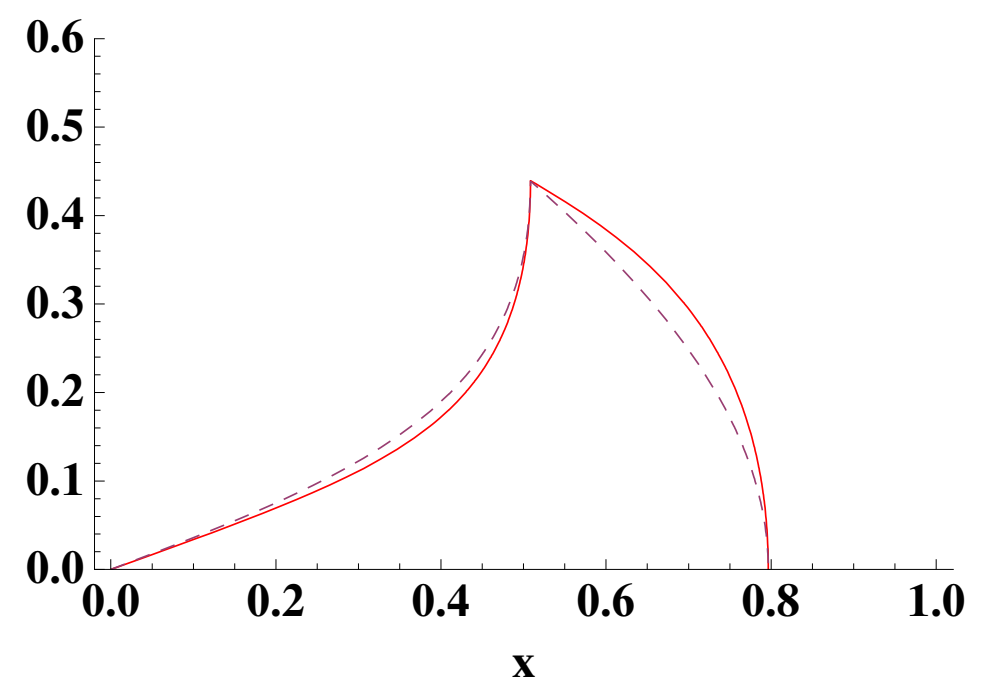

Figure 10. Unnormalized $p_{T}$ distributions for $a+b \rightarrow c+3, c \rightarrow 1+2$, assuming that the matrix element for $a+b \rightarrow c+3$ is constant (dashed curve) or is given by eq. (A.47) (solid curve). The rescaled transverse momentum is defined by $x \equiv 2 p_{T} / \sqrt{s}$ and can take on values in the range $0 \leq x \leq x_{\max }$, where $x_{\max } \equiv \frac{1}{2}(1-z)\left[1+y(1-z)+\lambda^{1 / 2}(1, y, y z)\right]$. The masses of particles $c$ and $d$ are fixed by $y \equiv M^{2} / s=0.5$ and $z \equiv m^{2} / M^{2}=0.1$. To facilitate the comparison of the two $p_{T}$ distributions, the relative normalization of the two curves has been fixed such that the peaks of the distributions coincide. The location of the peak is given by eq. (A.46) for both curves.

where $N$ is an overall dimensionless normalization factor that depends on the relevant couplings. The resulting unnormalized $p_{T}$ distribution is exhibited in figure 10. Note that the shape of the $p_{T}$ distribution is dominated by the explicit kinematic factors that appear in eq. (A.38), and depends quite weakly on the actual form of the squared-matrix element given in eq. (A.47). Moreover, the location of the peak in the $p_{T}$ distribution is unchanged and given by eq. (A.46), as a consequence of structure of the kinematic limits given in eqs. (A.35) and (A.36).

The location of the Jacobian peak given in eq. (A.46) depends implicitly on $\sqrt{s}$, which is the partonic center-of-mass energy in the above analysis. The differential cross section for the hadronic scattering process, $A+B \rightarrow c+3+X \rightarrow 1+2+3+X$ is obtained by convoluting the $p_{T}$ distribution of the partonic subprocess, $a+b \rightarrow c+3 \rightarrow 1+2+3$, with the product of the parton distribution functions $f_{a}^{A}\left(x_{1}, Q^{2}\right) f_{b}^{B}\left(x_{2}, Q^{2}\right)$, where the total centerof-mass squared-energy $S$ is related to the partonic center-of-mass energy via $s=x_{1} x_{2} S$, and $Q$ is the factorization scale. In the convolution, partonic center-of-mass energies close to the energy threshold for the partonic process provide the dominant contribution to the production of the final state. In this case, one can derive an approximate formula for the location of the Jacobian peak that does not depend on the partonic center of mass energy. The threshold for $a+b \rightarrow c+3$ corresponds to the point at which

$$
\lambda\left(s, M^{2}, m^{2}\right)=\left(s+M^{2}-m^{2}\right)^{2}-4 s M^{2}=0 .
$$


At this point $s+M^{2}-m^{2}=2 M \sqrt{s}$ (or equivalently, $\sqrt{s}=M+m$ ), in which case

$$
E_{1}^{-}=E_{1}^{+}=\frac{M^{2}-m^{2}}{2 M} \text {. }
$$

Of course, if $E_{1}^{-}=E_{1}^{+}$then the cross-section given in eq. (A.38) vanishes exactly at threshold. However, if we are close to threshold, eq. (A.49) still provides a decent approximation to $E_{1}^{-}$, in which case the location of the Jacobian peak is:

$$
\left(p_{T}\right)_{\text {peak }}=E_{1}^{-} \simeq \frac{M^{2}-m^{2}}{2 M}=\frac{1}{2} \xi M
$$

which is independent of the partonic center-of-mass energy.

In this paper, we have numerically computed the transverse momentum distribution of the hadronic scattering process, taking into account the partonic scattering process at all allowed values of the partonic center-of-mass energy. In particular, as the partonic centerof-mass energy is increased above the threshold energy for $a+b \rightarrow c+3$, the location of the peak of the partonic transverse momentum distribution, $E_{1}^{-}$[cf. eq. (A.26)] decreases relative to the estimate given in eq. (A.50). Thus, we expect the actual peak in the transverse momentum distribution of the hadronic scattering process (or equivalently in the missing transverse energy distribution) to be somewhat less than the result of eq. (A.50). This is indeed the case in the $\not{ }_{T}$ distributions that we exhibit in this paper.

Note that in the approximation that the transverse momentum of particle $c$ is due entirely from the hard scattering process (i.e. transverse momentum of the initial partons and the spectators are neglected), the distribution of the missing transverse energy (i.e. particles 2 and 3 of the hard scattering process) should precisely match that of the transverse momentum of the monojet (i.e. particle 1 of the hard scattering process). Of course, the effects of spectators, initial and final state radiation, fragmentation of final state partons, jet mismeasurements and detector effects will tend to reduce the sharpness of the peak in the $\not_{T}$ distributions as compared to that of figure 10 .

Open Access. This article is distributed under the terms of the Creative Commons Attribution Noncommercial License which permits any noncommercial use, distribution, and reproduction in any medium, provided the original author(s) and source are credited.

\section{References}

[1] Particle Data Group collaboration, K. Nakamura et al., Review of particle physics, J. Phys. G 37 (2010) 075021 [SPIRES].

[2] S.P. Martin, Dimensionless supersymmetry breaking couplings, flat directions and the origin of intermediate mass scales, Phys. Rev. D 61 (2000) 035004 [hep-ph/9907550] [SPIRES].

[3] ILC collaboration, G. Aarons et al., International Linear Collider reference design report. Volume 2: Physics at the ILC, arXiv:0709.1893 [SPIRES].

[4] LHC/LC Study Group collaboration, G. Weiglein et al., Physics interplay of the LHC and the ILC, Phys. Rept. 426 (2006) 47 [hep-ph/0410364] [SPIRES]. 
[5] A. Freitas and P.Z. Skands, Determining the SUSY-QCD Yukawa coupling, JHEP 09 (2006) 043 [hep-ph/0606121] [SPIRES].

[6] A. Freitas, P.Z. Skands, M. Spira and P.M. Zerwas, Examining the identity of Yukawa with gauge couplings in supersymmetric QCD at LHC, JHEP 07 (2007) 025 [hep-ph/0703160] [SPIRES].

[7] A. Brandenburg, M. Maniatis, M.M. Weber and P.M. Zerwas, Squarks and gluinos at a TeV $e^{+} e^{-}$collider: testing the identity of Yukawa and gauge couplings in SUSY-QCD, Eur. Phys. J. C 58 (2008) 291 [arXiv:0806 .3875] [SPIRES].

[8] S. Bornhauser, M. Drees, H.K. Dreiner and J.S. Kim, Rapidity gap events in squark pair production at the LHC, Phys. Rev. D 80 (2009) 095007 [arXiv:0909.2595] [SPIRES].

[9] UA1 collaboration, G. Arnison et al., Experimental observation of events with large missing

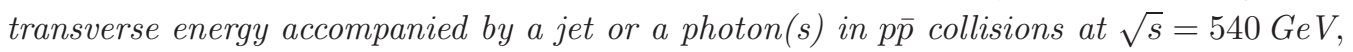
Phys. Lett. B 139 (1984) 115 [SPIRES].

[10] M. Gluck and E. Reya, Possible squark production at the proton-antiproton collider, Phys. Rev. Lett. 51 (1983) 867 [Erratum ibid. 51 (1983) 1307] [SPIRES].

[11] H.E. Haber and G.L. Kane, Signatures and possible evidence for supersymmetry at the CERN collider, Phys. Lett. B 142 (1984) 212 [SPIRES].

[12] J.R. Ellis and H. Kowalski, Gluino signatures at the pp collider, Phys. Lett. B 142 (1984) 441 [SPIRES].

[13] J.R. Ellis and H. Kowalski, Supersymmetric particles at the CERN pp collider, Nucl. Phys. B 246 (1984) 189 [SPIRES].

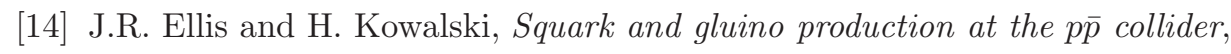
Nucl. Phys. B 259 (1985) 109 [SPIRES].

[15] J.R. Ellis and H. Kowalski, Can the gluino be light?, Phys. Lett. B 157 (1985) 437 [SPIRES].

[16] V.D. Barger, K. Hagiwara, J. Woodside and W.-Y. Keung, Possible supersymmetry scenario for $p \bar{p}$ collider monojet events and unaccompanied "photon" events, Phys. Rev. Lett. 53 (1984) 641 [SPIRES].

[17] V.D. Barger, K. Hagiwara and W.-Y. Keung, Constraints on squark masses from CERN $p \bar{p}$ collider data, Phys. Lett. B 145 (1984) 147 [SPIRES].

[18] A.R. Allan, E.W.N. Glover and A.D. Martin, Scalar quark signatures at the $\bar{p} p$ collider, Phys. Lett. B 146 (1984) 247 [SPIRES].

[19] N.D. Tracas and S.D.P. Vlassopulos, Prompt photino production at the CERN $\bar{p} p$ collider energies and beyond, Phys. Lett. B 149 (1984) 253 [SPIRES].

[20] V.D. Barger, K. Hagiwara, W.-Y. Keung and J. Woodside, Testing supersymmetry interpretations of anomalous missing $p_{T}$ events observed at the CERN p $\bar{p}$ collider, Phys. Rev. D 31 (1985) 528 [SPIRES].

[21] E. Reya and D.P. Roy, Have gluinos been observed at the p $\bar{p}$ collider?, Phys. Rev. Lett. 53 (1984) 881 [SPIRES].

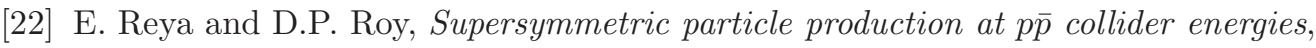
Phys. Rev. D 32 (1985) 645 [SPIRES].

[23] R.M. Barnett, H.E. Haber and G.L. Kane, Implications of a systematic study of the CERN monojets for supersymmetry, Phys. Rev. Lett. 54 (1985) 1983 [SPIRES]. 
[24] F. Delduc, H. Navelet, R.B. Peschanski and C.A. Savoy, Squark pair collisions mechanisms

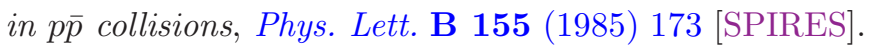

[25] M. Gluck, E. Reya and D.P. Roy, Gluinos and lower bounds for squark and slepton masses

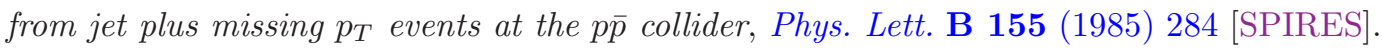

[26] A. De Rujula and R. Petronzio, Light gluinos and heavy squarks confront the collider data, Nucl. Phys. B 261 (1985) 587 [SPIRES].

[27] R.M. Barnett, H.E. Haber and G.L. Kane, Supersymmetry: lost or found?, Nucl. Phys. B 267 (1986) 625 [SPIRES].

[28] E. Reya and D.P. Roy, Constraints on gluino and squark masses from the new missing $p_{T}$ data at the $p \bar{p}$ collider, Phys. Lett. B 166 (1986) 223 [SPIRES].

[29] S.D. Ellis, R. Kleiss and W.J. Stirling, Monojets in the standard model, Phys. Lett. B 167 (1986) 464 [SPIRES].

[30] L. Vacavant and I. Hinchliffe, Signals of models with large extra dimensions in ATLAS, J. Phys. G 27 (2001) 1839 [SPIRES].

[31] CMS collaboration, L. Benucci, Search for extra dimensions in a single-jet and missing energy channel at CMS experiment, in Proceedings of the XXth Hadron Collider Physics Symposium, PoS (HCP2009) 065 [arXiv: 1001.5428] [SPIRES].

[32] T.G. Rizzo, Identification of the origin of monojet signatures at the LHC, Phys. Lett. B 665 (2008) 361 [arXiv:0805.0281] [SPIRES].

[33] The ATLAS collaboration, Early supersymmetry searches in channels with jets and missing transverse momentum with the ATLAS detector, ATLAS Note ATLAS-CONF-2010-065.

[34] D.S.M. Alves, E. Izaguirre and J.G. Wacker, It's on: early interpretations of ATLAS results in jets and missing energy searches, arXiv:1008.0407 [SPIRES].

[35] H. Baer, D.D. Karatas and X. Tata, Gluino and squark production in association with gauginos at hadron supercolliders, Phys. Rev. D 42 (1990) 2259 [SPIRES].

[36] G. Bozzi, B. Fuks, B. Herrmann and M. Klasen, Squark and gaugino hadroproduction and decays in non-minimal flavour violating supersymmetry, Nucl. Phys. B $\mathbf{7 8 7}$ (2007) 1 [arXiv: 0704.1826] [SPIRES].

[37] G.J. Gounaris, J. Layssac, P.I. Porfyriadis and F.M. Renard, Single neutralino production at CERN LHC, Phys. Rev. D 71 (2005) 075012 [hep-ph/0411366] [SPIRES].

[38] G. Bélanger, F. Boudjema, A. Pukhov and A. Semenov, Dark matter direct detection rate in a generic model with MicrOMEGAs2.1, Comput. Phys. Commun. 180 (2009) 747 [arXiv: 0803.2360] [SPIRES].

[39] L. Covi, M. Olechowski, S. Pokorski, K. Turzynski and J.D. Wells, Supersymmetric mass spectra for gravitino dark matter with a high reheating temperature, JHEP 01 (2011) 033 [arXiv: 1009.3801] [SPIRES].

[40] G.F. Giudice, T. Han, K. Wang and L.-T. Wang, Nearly degenerate gauginos and dark matter at the LHC, Phys. Rev. D 81 (2010) 115011 [arXiv: 1004.4902] [SPIRES].

[41] M. Carena, A. Freitas and C.E.M. Wagner, Light stop searches at the LHC in events with one hard photon or jet and missing energy, JHEP 10 (2008) 109 [arXiv:0808.2298] [SPIRES].

[42] J. Goodman et al., Constraints on light Majorana dark matter from colliders, Phys. Lett. B 695 (2011) 185 [arXiv: 1005.1286] [SPIRES]. 
[43] Y. Bai, P.J. Fox and R. Harnik, The Tevatron at the frontier of dark matter direct detection, JHEP 12 (2010) 048 [arXiv: 1005.3797] [SPIRES].

[44] J. Goodman et al., Constraints on dark matter from colliders, arXiv:1008.1783 [SPIRES].

[45] J. Goodman et al., Gamma ray line constraints on effective theories of dark matter, Nucl. Phys. B 844 (2011) 55 [arXiv: 1009.0008] [SPIRES].

[46] M. Beltrán, D. Hooper, E.W. Kolb, Z.A.C. Krusberg and T.M.P. Tait, Maverick dark matter at colliders, JHEP 09 (2010) 037 [arXiv: 1002 .4137] [SPIRES].

[47] M. Klasen and G. Pignol, New results for light gravitinos at hadron colliders: Tevatron limits and LHC perspectives, Phys. Rev. D 75 (2007) 115003 [hep-ph/0610160] [SPIRES].

[48] H.K. Dreiner et al., Rare meson decays into very light neutralinos, Phys. Rev. D 80 (2009) 035018 [arXiv:0905.2051] [SPIRES].

[49] T. Hebbeker, Can the sneutrino be the lightest supersymmetric particle?, Phys. Lett. B 470 (1999) 259 [hep-ph/9910326] [SPIRES].

[50] H. Baer, K.-m. Cheung and J.F. Gunion, A heavy gluino as the lightest supersymmetric particle, Phys. Rev. D 59 (1999) 075002 [hep-ph/9806361] [SPIRES].

[51] S. Raby and K. Tobe, The phenomenology of SUSY models with a gluino LSP, Nucl. Phys. B 539 (1999) 3 [hep-ph/9807281] [SPIRES].

[52] J.R. Ellis, J.S. Hagelin, D.V. Nanopoulos, K.A. Olive and M. Srednicki, Supersymmetric relics from the big bang, Nucl. Phys. B 238 (1984) 453 [SPIRES].

[53] J.F. Gunion and H.E. Haber, Two-body decays of neutralinos and charginos, Phys. Rev. D 37 (1988) 2515 [SPIRES].

[54] G.L. Kane, C.F. Kolda, L. Roszkowski and J.D. Wells, Study of constrained minimal supersymmetry, Phys. Rev. D 49 (1994) 6173 [hep-ph/9312272] [SPIRES].

[55] L. Randall and R. Sundrum, Out of this world supersymmetry breaking, Nucl. Phys. B 557 (1999) 79 [hep-th/9810155] [SPIRES].

[56] G.F. Giudice, M.A. Luty, H. Murayama and R. Rattazzi, Gaugino mass without singlets, JHEP 12 (1998) 027 [hep-ph/9810442] [SPIRES].

[57] T. Gherghetta, G.F. Giudice and J.D. Wells, Phenomenological consequences of supersymmetry with anomaly-induced masses, Nucl. Phys. B 559 (1999) 27 [hep-ph/9904378] [SPIRES].

[58] T. Moroi and L. Randall, Wino cold dark matter from anomaly-mediated SUSY breaking, Nucl. Phys. B 570 (2000) 455 [hep-ph/9906527] [SPIRES].

[59] L. Vacavant and I. Hinchliffe, Model independent extra-dimension signatures with ATLAS, hep-ex/0005033 [SPIRES].

[60] The ATLAS collaboration, G. Aad et al., Expected performance of the ATLAS experiment - detector, trigger and physics, arXiv:0901.0512 [SPIRES].

[61] A.J. Barr and C. Gwenlan, The race for supersymmetry: using mT2 for discovery, Phys. Rev. D 80 (2009) 074007 [arXiv:0907.2713] [SPIRES].

[62] S. Dawson, E. Eichten and C. Quigg, Search for supersymmetric particles in hadron-hadron collisions, Phys. Rev. D 31 (1985) 1581 [SPIRES].

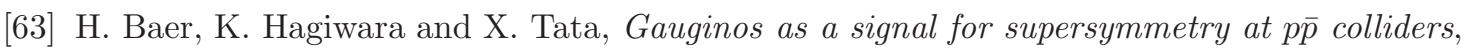
Phys. Rev. D 35 (1987) 1598 [SPIRES].

[64] A.J. Barr, C.G. Lester, M.A. Parker, B.C. Allanach and P. Richardson, Discovering anomaly-mediated supersymmetry at the LHC, JHEP 03 (2003) 045 [hep-ph/0208214] [SPIRES]. 
[65] L. Lönnblad, Development strategies for PYTHIA version 7, Comput. Phys. Commun. 118 (1999) 213 [hep-ph/9810208] [SPIRES].

[66] M. Bahr et al., HERWIG++ physics and manual, Eur. Phys. J. C 58 (2008) 639 [arXiv: 0803.0883] [SPIRES].

[67] M. Bahr, S. Gieseke and M.H. Seymour, Simulation of multiple partonic interactions in HERWIG++, JHEP 07 (2008) 076 [arXiv:0803.3633] [SPIRES].

[68] http://projects.hepforge.org/herwig/trac/ticket/317.

[69] M. Dobbs and J.B. Hansen, The HepMC C++ Monte Carlo event record for high energy physics, Comput. Phys. Commun. 134 (2001) 41 [SPIRES].

[70] R. Brun and F. Rademakers, ROOT: an object oriented data analysis framework, Nucl. Instrum. Meth. A 389 (1997) 81 [SPIRES].

[71] P. Canal, R. Brun, V. Fine, L. Janyst, J. Lauret and P. Russo, Support for significant evolutions of the user data model in ROOT files, J. Phys. Conf. Ser. 219 (2010) 032004 [SPIRES].

[72] http://www.lpthe.jussieu.fr/ ${ }^{\sim}$ salam/fastjet/.

[73] M. Cacciari, G.P. Salam and G. Soyez, The anti- $k_{t}$ jet clustering algorithm, JHEP 04 (2008) 063 [arXiv:0802.1189] [SPIRES].

[74] A.H. Chamseddine, R.L. Arnowitt and P. Nath, Locally supersymmetric grand unification, Phys. Rev. Lett. 49 (1982) 970 [SPIRES].

[75] L. Álvarez-Gaumé, M. Claudson and M.B. Wise, Low-energy supersymmetry, Nucl. Phys. B 207 (1982) 96 [SPIRES].

[76] L.E. Ibáñez, Locally supersymmetric SU(5) grand unification, Phys. Lett. B 118 (1982) 73 [SPIRES].

[77] R. Barbieri, S. Ferrara and C.A. Savoy, Gauge models with spontaneously broken local supersymmetry, Phys. Lett. B 119 (1982) 343 [SPIRES].

[78] N. Ohta, Grand unified theories based on local supersymmetry, Prog. Theor. Phys. 70 (1983) 542 [SPIRES].

[79] L.J. Hall, J.D. Lykken and S. Weinberg, Supergravity as the messenger of supersymmetry breaking, Phys. Rev. D 27 (1983) 2359 [SPIRES].

[80] S.K. Soni and H.A. Weldon, Analysis of the supersymmetry breaking induced by $N=1$ supergravity theories, Phys. Lett. B 126 (1983) 215 [SPIRES].

[81] B.C. Allanach, SOFTSUSY: a program for calculating supersymmetric spectra, Comput. Phys. Commun. 143 (2002) 305 [hep-ph/0104145] [SPIRES].

[82] P.Z. Skands et al., SUSY Les Houches Accord: interfacing SUSY spectrum calculators, decay packages and event generators, JHEP 07 (2004) 036 [hep-ph/0311123] [SPIRES].

[83] CDF collaboration, T. Aaltonen et al., Inclusive search for squark and gluino production in $p \bar{p}$ collisions at $\sqrt{s}=1.96 \mathrm{TeV}$, Phys. Rev. Lett. 102 (2009) 121801 [arXiv:0811.2512] [SPIRES].

[84] D0 collaboration, V.M. Abazov et al., Search for squarks and gluinos in events with jets and missing transverse energy using $2.1 \mathrm{fb}^{-1}$ of $p \bar{p}$ collision data at $\sqrt{s}=1.96 \mathrm{TeV}$, Phys. Lett. B 660 (2008) 449 [arXiv:0712.3805] [SPIRES].

[85] R. Hauser, The ATLAS trigger system, Eur. Phys. J. C 34 (2004) S173 [SPIRES].

[86] CMS collaboration, D. Giordano, The CMS high-level trigger selection, Nucl. Phys. Proc. Suppl. 150 (2006) 299 [SPIRES]. 
[87] K. Desch, S. Fleischmann, P. Wienemann, H.K. Dreiner and S. Grab, Stau as the lightest supersymmetric particle in R-parity violating SUSY models: discovery potential with early LHC data, arXiv: 1008.1580 [SPIRES].

[88] Review of the ATLAS technical design report on the forward detectors for the measurement of elastic scattering and luminosity, CERN-LHCC-2008-013 [SPIRES].

[89] I. Efthymiopoulos, Elastic cross-section and luminosity measurement in ATLAS at LHC, hep-ex/0510078 [SPIRES].

[90] M. Boonekamp, Luminosity measurement in ATLAS, DAPNIA-04-231 [SPIRES].

[91] J. Pumplin et al., New generation of parton distributions with uncertainties from global QCD analysis, JHEP 07 (2002) 012 [hep-ph/0201195] [SPIRES].

[92] A.D. Martin, W.J. Stirling, R.S. Thorne and G. Watt, Parton distributions for the LHC, Eur. Phys. J. C 63 (2009) 189 [arXiv:0901.0002] [SPIRES].

[93] M. Gluck, P. Jimenez-Delgado and E. Reya, Dynamical parton distributions of the nucleon and very small-x physics, Eur. Phys. J. C 53 (2008) 355 [arXiv:0709.0614] [SPIRES].

[94] M. Gluck, P. Jimenez-Delgado, E. Reya and C. Schuck, On the role of heavy flavor parton distributions at high energy colliders, Phys. Lett. B 664 (2008) 133 [arXiv:0801.3618] [SPIRES].

[95] A. Sherstnev and R.S. Thorne, Parton distributions for LO generators, Eur. Phys. J. C 55 (2008) 553 [arXiv:0711.2473] [SPIRES].

[96] T. Plehn, Measuring the MSSM Lagrangean, Czech. J. Phys. 55 (2005) B213 [hep-ph/0410063] [SPIRES].

[97] Prospino 2.1, http://www.thphys.uni-heidelberg.de/ ${ }^{\sim}$ plehn/prospino.

[98] H. Baer, V. Barger, A. Lessa and X. Tata, Capability of LHC to discover supersymmetry with $\sqrt{s}=7 \mathrm{TeV}$ and $1 \mathrm{fb}^{-1}$, JHEP 06 (2010) 102 [arXiv: 1004.3594] [SPIRES].

[99] J.L. Feng and T. Moroi, Supernatural supersymmetry: phenomenological implications of anomaly-mediated supersymmetry breaking, Phys. Rev. D 61 (2000) 095004 [hep-ph/9907319] [SPIRES].

[100] K. Huitu, J. Laamanen and P.N. Pandita, Sparticle spectrum and constraints in anomaly mediated supersymmetry breaking models, Phys. Rev. D 65 (2002) 115003 [hep-ph/0203186] [SPIRES].

[101] P. Bechtle, K. Desch, M. Uhlenbrock and P. Wienemann, Constraining SUSY models with Fittino using measurements before, with and beyond the LHC,

Eur. Phys. J. C 66 (2010) 215 [arXiv:0907.2589] [SPIRES].

[102] J.A. Aguilar-Saavedra et al., Supersymmetry parameter analysis: SPA convention and project, Eur. Phys. J. C 46 (2006) 43 [hep-ph/0511344] [SPIRES].

[103] V.D. Barger and R.J.N. Phillips, Collider physics, Westview Press, Boulder U.S.A. (1996).

[104] E. Byckling and K. Kajantie, Particle kinematics, John Wiley \& Sons Ltd., London U.K. (1973).

[105] P. Nyborg, H.S. Song, W. Kernan and R.H. Good Jr., Phase-space considerations for four-particle final states, Phys. Rev. 140 (1965) B914.

[106] P. Nyborg, Phase-space considerations for five-particle final states, Phys. Rev. 140 (1965) B921. 